\title{
Gut microbiota in human metabolic health and disease
}

\author{
Yong Fan (1) and Oluf Pedersen (10)
}

Abstract $\mid$ Observational findings achieved during the past two decades suggest that the intestinal microbiota may contribute to the metabolic health of the human host and, when aberrant, to the pathogenesis of various common metabolic disorders including obesity, type 2 diabetes, non-alcoholic liver disease, cardio-metabolic diseases and malnutrition. However, to gain a mechanistic understanding of how the gut microbiota affects host metabolism, research is moving from descriptive microbiota census analyses to cause-and-effect studies. Joint analyses of high-throughput human multi-omics data, including metagenomics and metabolomics data, together with measures of host physiology and mechanistic experiments in humans, animals and cells hold potential as initial steps in the identification of potential molecular mechanisms behind reported associations. In this Review, we discuss the current knowledge on how gut microbiota and derived microbial compounds may link to metabolism of the healthy host or to the pathogenesis of common metabolic diseases. We highlight examples of microbiota-targeted interventions aiming to optimize metabolic health, and we provide perspectives for future basic and translational investigations within the nascent and promising research field.

From the outcome of recent epidemiological, physiological and omics-based studies, complemented by cellular studies and experiments in animals, it appears that a considerable part of the environmental influence on human health and disease risk may be mediated or modified by microbial communities ${ }^{1}$. These microorganisms, collectively referred to as the microbiota, include a vast number of interacting bacteria, archaea, bacteriophages, eukaryotic virus and fungi coexisting on human surfaces and in all body cavities ${ }^{1}$. The majority of them are commensal or mutualistic microorganisms ${ }^{1}$. The collection of all intestinal microbial genes (that is, the microbiome) in an individual represents a genetic repertoire that is more than one order of magnitude higher in genes than the human genome ${ }^{1}$. The majority of microorganisms that inhabit humans reside within the intestines and are influenced by mode of birth, infant feeding, lifestyle, medication and the genetics of the host. The gut microbiome has important roles in the training of host immunity, digesting food, regulating gut endocrine function and neurological signalling, modifying drug action and metabolism, eliminating toxins and producing numerous compounds that influence the host ${ }^{1}$.

Here, we review the literature on the intestinal microbiota of the human host with a focus on bacteria and archaea, and their potential importance for human metabolism. In the literature, there is no consensus on how to define metabolic health. Yet a tentative definition is to have an overall metabolism (as estimated from an array of various measures of body organ functions, such as those of the liver, and intestinal, fat, muscle, heart and brain tissues) that in representative epidemiological studies is linked with both desirable life quality and longevity. A considerable part of the world population, however, primarily due to overeating of processed energy-dense food, urbanization with sedentary occupations and leisure-time inactivity often combined with smoking addiction, has a suboptimal or even a poor metabolic health. The implications are a high prevalence of metabolic disorders, including obesity, non-alcoholic liver disease, type 2 diabetes mellitus (T2D) as well as cardio-metabolic disease (CMD), and premature death $^{2-6}$. In some developing countries, the metabolic health landscape is quite the opposite - a major part of the population is chronically malnourished ${ }^{7}$.

Despite the immense differences in pathologies of common chronic metabolic disorders, they are associated with shared and disease-specific abnormalities in the composition and function of the intestinal microbiota $^{8-11}$. Since the start of the microbiota research era, whether disease-associated aberrant microbiota are involved in disease causation (that is, predisposition, initiation or progression) or are secondary phenomena has been widely debated. However, one of the first pieces of evidence for a mechanistic involvement of the gut microbiota in the regulation of metabolism was provided in 


\section{Box 1 | Tools for dissecting gut microbiota-metabolic health associations}

\begin{abstract}
Currently, there is major interest in exploring the potential role of the intestinal microbiome and linked faecal, blood and urine metabolomes as determinants for metabolic health and various metabolic diseases. Recent advances in shotgun sequencing technologies and a reduction in sequencing costs together with advances in bioinformatics have made it possible to capture a more comprehensive view of the entire community of gut microorganisms and their functional potentials. Similarly, various sensitive and precise technologies for profiling the metabolome of body fluids are available. Here, we summarize some of the recent strategies that have been applied to uncover relationships between the gut microbiome and various metabolomes.
\end{abstract}

\section{Microbiome-wide association studies}

The microbiome-wide association study approach largely mimics genome-wide association studies identifying genetic variants in casecontrol studies that are associated with a phenotype, often a disease state. In the case of microbiome-wide association studies, microbial DNA is purified from intestinal or faecal samples and subjected to deep shotgun-based sequencing. Individually, the identified and assembled genes are then integrated to construct a cross-sampled, non-redundant microbial gene catalogue. The abundance of each gene is determined by counting the reads of matching sequences in the individual sample.

Applying various bioinformatic algorithms and correlation coefficients, assembled genes in the microbiome data are clustered into specific groups, such as metagenomic linkage groups $^{8}$, metagenomic species ${ }^{62}$, co-abundance gene groups ${ }^{168}$ or metagenomic species pan-genomes ${ }^{169}$. Sequence reads from individual samples that map to the metagenomic linkage groups, metagenomic species, co-abundance gene groups, metagenomic species pan-genomes and their contigs, respectively, are then extracted and assembled into high-quality draft genomes, each of which is defined as a species or a group of highly related species. Recently, it has also become possible to define strain-level signatures in gut microbiomes. These tools include StrainPhlAn that is based on applying single-nucleotide variant analysis to the core genome of sequenced microbial isolate ${ }^{170}$, MetaMLST that is based on speciesspecific hypervariable loci ${ }^{171}$ and MetaPanPhlAn that is based on pangenome profiling ${ }^{172}$. However, these methods rely on the coverage and quality of reference genomes ${ }^{173}$. Results from strain-level profiling still need to be validated by obtaining a pure culture containing the target bacterial isolate. In addition, associations with health or disease status can be characterized for individual microbial genes, taxa or modules of microbial functional potentials ${ }^{14,17,45}$. Classifiers such as supervised machine learning or random forest can be used to assign each sample to a certain category. To validate associations identified by microbiome-wide association studies, additional metagenomics data sets such as samples from other independent studies are included.

\section{Integrated analyses of microbiome and linked metabolomes} Gut bacteria can produce various bioactive metabolites, which can enter the bloodstream of the host through absorption into the enterohepatic circulation. Specific metabolites associated with a disease phenotype can be identified by mass spectrometry or nuclear magnetic resonance-based metabolomics of faeces, plasma, urine or other biofluids, making it possible to conduct joint analyses of the microbiome, metabolome and host phenotypes to identify potential mechanistic links ${ }^{15}$. The analysis framework includes at least four steps: first, as described above, clustering of microbial genes, the functional potential of which can be constructed by organizing the genes into Kyoto Encyclopedia of Genes and Genomes functional modules based on sequence similarity to proteins with known functional characteristics ${ }^{15}$. The second step involves clustering of metabolites showing co-abundance to reduce dimension of metabolomics features, given that metabolites are often shaped by common pathways that could be regulated by precursors ${ }^{14}$. By using weighted gene co-expression network analysis, which was originated from gene expression analysis, co-abundant metabolites can be clustered ${ }^{174}$. Of note, one of the advantages of this algorithm is the use of a bin cluster for unannotated metabolites instead of clustering all metabolites. The next step is to apply non-parametric statistical tests to correlated clustered genes and metabolites. Finally, based on the hypothesis that microbial functional correlations could be driven by one or few species or by a group of species, it is recommended to perform leave-one-out cross-validation to evaluate the contribution of each gene cluster by removing it from the associations ${ }^{14}$. Correlation analysis between these specific metabolites and prevalent gene clusters may lead to the identification of specific species that harbour the genes needed to produce precursors of the disease-relevant metabolites that are produced either by the gut microbiota or by both the host and the gut microbiota ${ }^{14,45}$. Eventually, identified microbial candidates are evaluated in rodent models (BOX 2).
Supervised machine learning A form of applied artificial intelligence where the algorithm learns by experience to classify new data according to prior labels.

Random forest

A machine learning approach where a multitude of algorithms (decision trees) are used to optimize, for instance, classification or regression of data sets.

Kyoto Encyclopedia of Genes and Genomes

A publicly available database in bioinformatics and system medicine-driven analyses with information on omics-generated data, biological pathways, chemicals and drugs
2004 when it was shown in mice that the gut microbiota regulates host capacity for harvesting energy from the diet as well as energy storage ${ }^{12}$. Subsequently, and spearheaded by application of next-generation microbiome sequencing, generation of comprehensive microbial gene catalogues, development of targeted bioinformatics and availability of high data storage and operational capacity, the research field has delivered an enormous amount of new knowledge about the potential role of the gut microbiota in metabolism ${ }^{13-15}$ (BOX 1).

In this Review, we discuss taxonomic and functional characteristics of the intestinal microbiota in the metabolically healthy human host and the implication of a reduction in gut microbial diversity for host metabolism. This is followed by highlighting aspects of abnormal gut microbiota composition and function, and the relationship to dysmetabolism in obesity, T2D, CMD, metabolic liver disease and malnutrition. We evaluate a series of recently discovered gut microbiota-derived compounds and their potential role as messengers between the gut microbiota and host metabolism in physiological and diseased states. We highlight examples of microbiota-based therapies to improve metabolic health and, finally, we outline potential avenues for future epidemiological and experimental studies (BOX 2 and BOX 3).

\section{Healthy gut microbiota}

A fundamental assumption for claiming disruption of gut microbiota in states of metabolic disorders is knowledge on the composition and function of the gut microbiota of metabolically healthy people. However, a healthy human gut microbiota has not been defined at any profound taxonomic resolution. The relative distribution of gut bacteria and archaea is unique to an individual, partly owing to strain-level diversities and differences in microbial growth rates ${ }^{16}$ and in structural variants within the microbial genes ${ }^{17,18}$, and partly owing to influence from the considerable inter-individual variation in environmental exposures and host genetics ${ }^{19}$. Overall, however, a high taxa diversity, high microbial gene richness and stable microbiome functional cores characterize healthy gut microbial communities ${ }^{18}$. Yet it is worth noting that high gut bacterial diversity and richness alone are not unbiased indicators of a healthy microbiota, because the intestinal transit time affects microbial richness ${ }^{20}$. A prolonged transit time may result in an increased richness but not necessarily in a healthy gut microbiota. 


\section{Box 2 |A framework to study causality of gut microbial components}

To progress from microbiome-disease associations to functions of the microbiome in a given disease, additional experiments are suggested to explore potential causality between microbial components and the disease of interest. Biological samples from affected and control individuals are analysed using shotgun sequencing and untargeted (that is, discovery-based and global but semi-quantitative) metabolomics profiling to identify microbial features or molecules differently abundant in various biofluids (see the figure, part a). In an untargeted run, the analyte is characterized by its retention time and its mass to charge ratio ( $\mathrm{m} / \mathrm{z}$ value). The identification of a novel compound is confirmed by generation of a nuclear magnetic resonance spectrum or matching fragmental behaviour and retention time to synthetic reference standards $s^{175}$. Both microbiome and metabolome data sets are subjected to integrative analysis to capture candidate biosynthetic gene clusters (BGCs), bacteria or communities that are highly related to metabolites. To identify whether the compound of interest is produced in a microbiota-dependent manner, triple quadrupole mass spectrometry can be used to perform targeted (that is, validation of well-defined metabolites often in a quantitative manner) profiling of samples from both germ-free and control mice (see the figure, part b). In addition, the conventionalization of antibiotic-treated mice to rescue gut microbiota can also specifically demonstrate the role of gut microbiota in generating specific compounds. When employing reaction-selecting modes in targeted capturing of the candidate analytes, both the first quadrupole (Q1) and the last quadrupole (Q3) are set at a specific mass, allowing only distinct monitoring of the ion fragment from a certain precursor, which results in an increased detecting sensitivity.

When the selected reaction monitoring is applied to multiple product ions from one or more precursor ions, the pattern is called multiple reaction monitoring ${ }^{176}$.

Human intestinal microbiome data and microbial-derived compounds can be associated to select candidate BGCs, microbial species or a microbial community whose presence correlates with the production of specific compounds (see the figure, part c). Dimensionality reduction is achieved by clustering genes that encode the biosynthetic enzymes for secondary metabolite pathways, binning co-abundant genes and assembling co-occurring microbes that are present in a defined ecological habitat in space and time $^{14,177}$.

Phenotypic profiling of germ-free mice harbouring a reference BGC, microbial species or microbial community can be profiled in a comparative targeted manner to examine the abundance changes of the compound of interest ${ }^{177}$.

To identify downstream signalling pathways that microbial-derived compounds may be involved in, a genetically altered mouse can be generated to see the change of microorganism-generated compound because of the inactivated downstream effects (see the figure, part d). The evidence collected above may stimulate to develop microbiota-directed interventions (BOX 3).

HOMA-IR, homeostatic model assessment of insulin resistance. Part c of figure adapted from REF. ${ }^{14}$, Springer Nature Limited. b
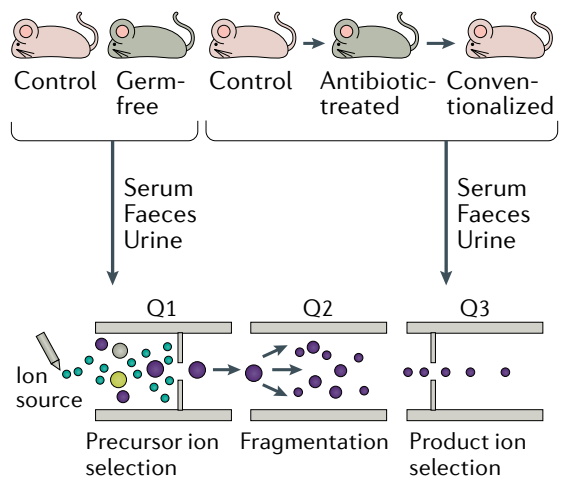

Targeted metabolomics

Control Antibiotic- Conventreated tionalized

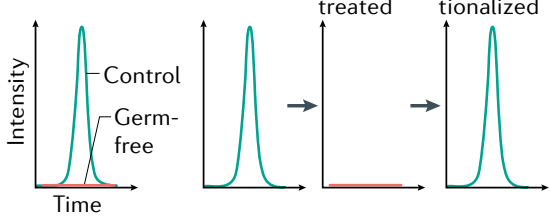

Microbiota-dependent fashion

Gene knockout

Control

Colonization of
germ-free mice
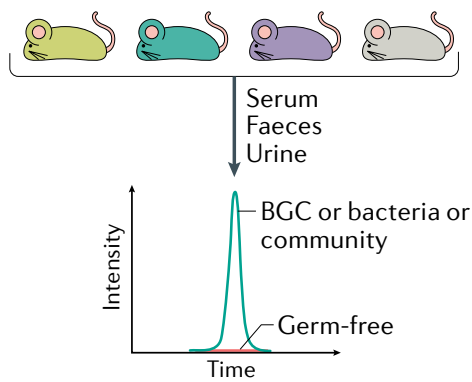

Comparative targeted metabolomics
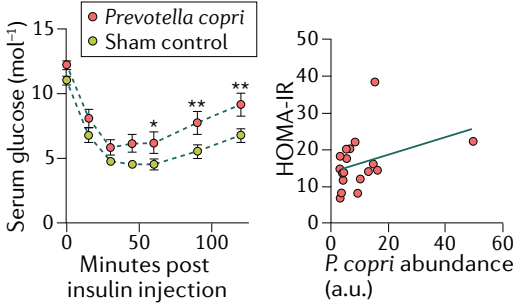

Phenotypic profiling

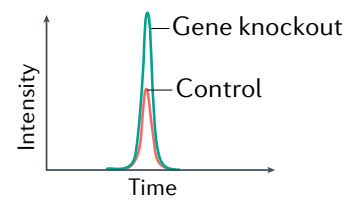

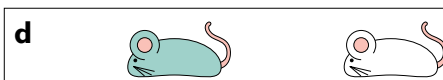

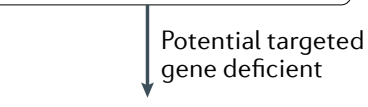

Microbial-based drug design

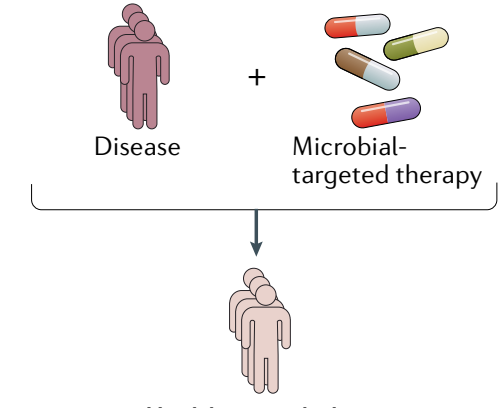

Healthy metabolism 
The mode of birth and access to breastfeeding shape the infant gut microbiota, and it matures gradually during childhood in response to environmental exposures $^{21,22}$. Thereafter, the intestinal microbiota remains relatively stable in late childhood, adolescence and adulthood until a decline in diversity occurs at advanced age, likely owing, in part, to a change in immune functions ${ }^{22}$. The mature healthy intestinal

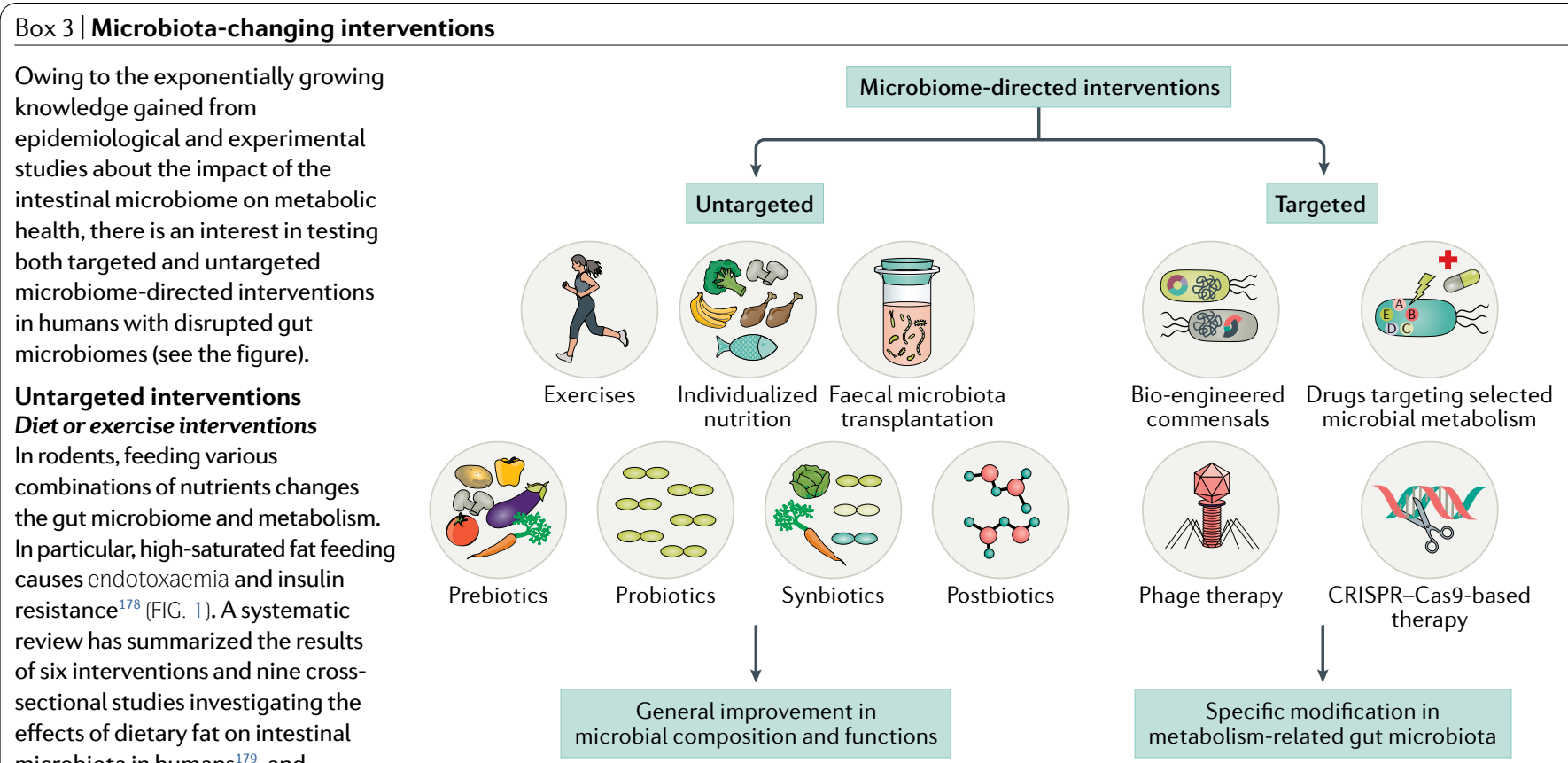

microbiota in humans ${ }^{179}$, and

demonstrated that diets high in saturated or monounsaturated fat negatively influenced the microbiota whereas diets high in polyunsaturated fat appeared to be neutral with respect to the microbiota. Similarly, high-polysaccharide diet interventions have resulted in altered gut microbiota linked with increased faecal, serum or urine concentrations of short-chain fatty acids, weight loss and improvements of cytokine and metabolome profiles ${ }^{180-183}$. Likewise, interventions with increased physical activity have shown adaptive and, in some cases, transmissible changes of the intestinal microbiome linked with an increased capacity for breakdown of lactate, branched-chain amino acids, an increased potential for synthesis of short-chain fatty acids and improvements of cardiorespiratory fitness and insulin sensitivity.

\section{Trials testing probiotics, prebiotics or postbiotics}

Bifidobacterium, Lactobacillus and Saccharomyces spp. have a long history as safe probiotics ${ }^{184}$. In addition, potential next-generation probiotics, which are not yet marketed, include Faecalibacterium prausnitzii ${ }^{185}$, Akkermansia muciniphila ${ }^{186}$ and several Clostridia spp. ${ }^{187}$. Systematic reviews of short-term randomized controlled trials in healthy individuals show no consistent effect of probiotics on gut microbial composition ${ }^{188}$. However, it is possible that some probiotics, for example A. muciniphila strains, do not even need to colonize the intestine to provide beneficial metabolic health effects ${ }^{189,190}$. Some non-digestible polysaccharides called prebiotics change the relative abundance of fermenting microbiota in the colon of rodents and induce improved gut barrier functions and in some cases improved metabolism ${ }^{191}$. Of note, randomized controlled trials testing various prebiotics have demonstrated that inulin-type fructans changed the gut microbiota composition in adult women with obesity, leading to modest changes in host metabolism ${ }^{192}$. In children who are overweight or have obesity, it is reported that oligofructose-enriched inulin prebiotics alter the intestinal microbiota and modestly reduce body weight, adiposity and inflammatory markers ${ }^{193}$. The same holds for the combination of various probiotic strains and prebiotics often named synbiotics. Postbiotics represent the pasteurized version of probiotics or parts of microbial strains possessing health-promoting effects ${ }^{194}$. A pilot trial of pasteurized A. muciniphila and its membrane protein Amuc_1100 demonstrated positive effects on markers of human metabolism ${ }^{186,195}$.

Heterologous and autologous faecal microbiota transplantation It is currently unsettled whether heterologous faecal microbiota transplantation will be an option in preventing or treating metabolic dysfunctions with aetiologies far more complex than chronic infections caused by Clostridioides difficile ${ }^{196}$. Challenges include lack of knowledge about the importance of dieting for stool graft colonization and survival, optimal anaerobic handling of donor faeces, immunological compatibility between donor and recipient, and the role of bacteriophages and fungi for a successful faecal microbiota transplantation. In parallel, there is a need for examining the feasibility and efficacy of the much less complicated autologous faecal microbiota transplantation stored prior to development of a metabolic disease, that is, faecal samples biobanked in childhood or adolescence.

\section{Targeted interventions}

\section{Bio-engineered commensals and drugs}

In mice, gavaging engineered Escherichia coli overexpressing the satiety factor $\mathrm{N}$-acylphosphatidylethanolamine alleviated high-fat diet-induced obesity, insulin resistance and hepatosteatosis ${ }^{197}$; and in rats with diabetes, a genetically modified Lactobacillus gasseri strain with the ability to express and secrete glucagon-like peptide 1 (GLP-1) increased insulin release and reduced hyperglycaemia ${ }^{198}$. Whether delivering genetically modified organisms carrying microbial genes to the human gut is acceptable for health and drug authorities or consumers is at present unsettled. Targeting specific microbial-synthesized metabolites by delivering tailored drugs is another emerging potential frontier to optimize metabolism. A prime example is the specific drug blockade of the microbial production of trimethylamine ${ }^{78}$.

\section{Bacteriophage therapy and CRISPR-Cas9 editing}

As most bacteriophages and archaeal viruses are specific to bacterial and archaeal strains, respectively, they may in future experiments be applied to target dysbiotic parts of the microbiome in individuals with metabolic disorders ${ }^{199}$. Similarly, it is at present unknown whether CRISPR-Cas can be applied to edit and reset dysbiotic parts of a human gut microbiome without causing undesired effects for the host $^{200}$. 
Dysmetabolism

Metabolic dysfunctions often including abdominal obesity, dyslipidaemia and higher blood glucose and higher blood pressure than normal.

Endotoxaemia

The presence of endotoxin(s) within the blood, for example bacterial lipopolysaccharides

Dyslipidaemia

An abnormal amount and an abnormal relative distribution of various lipids in the blood. microbial core functions include genes encoding glycosaminoglycan degradation, the production of short-chain fatty acids (SCFAs) via fermentation of complex polysaccharides and synthesis of specific lipopolysaccharides (LPS), and the biosynthesis of some essential amino acids and vitamins $\mathbf{s}^{13,18,23,24}$ (FIG. 1).

In summary, a 'gold standard' reference of a human gut microbiota with the capacity to promote host metabolic health does not exist. This is owing to a tremendous variation, especially at taxonomy levels, between individuals of different demography, ethnicity, sex, age and health status. The implications of this variation are that epidemiological studies must include controls for contextual factors or adjust for the contextual differences.

\section{Receding gut microbial diversity}

Compared with people living traditional lifestyles (for instance, people in East Africa or in the Amazonas) who have not been exposed to antimicrobial factors of modern living, most populations in technically developed countries of the world show reduced gut microbial diversity ${ }^{25}$. In parallel with urbanization, sewerage, higher standards of housing and improved hygiene in general, the abundance of Bacteroides, Prevotella, Desulfovibrio, Lactobacillus and Oxalobacter genera in the gut microbiota is declining ${ }^{25-27}$. This decline in diversity relates to a rise in the prevalence of common chronic metabolic disorders ${ }^{28}$. Similarly, in both lean individuals and individuals with obesity, low microbial gene richness links with a relative increase in adiposity, insulin resistance, inflammation and dyslipidaemia ${ }^{28}$. The precise and specific causes of the receding microbial richness and diversity in developed countries are elusive. However, besides the general improvement in standards of living and hygiene, the use of antibiotics to combat infectious diseases is suspected to be a contributor ${ }^{29}$. For instance, the use of antibiotics before pregnancy, during pregnancy or in early childhood may change the composition of the gut microbiota of infants and children, practices that have occurred in parallel with increases in the incidence of early-onset obesity ${ }^{30,31}$. In adults, short-term treatment of young healthy individuals with broad-spectrum antibiotics causes the long-term depletion of some commensal and mutualistic bacteria ${ }^{32}$. However, causal relationships between antibiotic use, disruption of the gut microbiota and dysmetabolism have not been shown ${ }^{33-35}$. The effects of antibiotics on the gut microbiota and metabolism may be dependent on host genetics because studies in mice fed a high-fat diet demonstrate that the effect of antibiotics on numerous metabolic variables is dependent on the genetic background of the animals ${ }^{36}$. Below, we discuss aspects of abnormal gut microbiota and the relationships to metabolism in obesity, T2D, metabolic liver disease and CMD, where the diversity of the gut microbiota of the affected is often reported to be reduced compared with healthy individuals, and even more so compared with people living traditional lifestyles ${ }^{28,37}$. In addition, we discuss how severe malnutrition during infancy and early childhood relates to a relative depletion of certain bacteria in the gut microbiota. In the absence of clinically controlled interventions aimed at restoring the richness and diversity of gut microbial communities in chronic disorders, it is not possible to evaluate whether a receding intestinal microbiota is part of disease causation or a secondary adaptation in states of chronic non-communicable disorders. Yet there is some evidence that dietary interventions may improve low microbial gene richness and host metabolism in individuals with obesity ${ }^{38}$.

\section{Gut microbiota of metabolic pathologies}

Gut microbiota is aberrant in obesity. The incidence of obesity and its metabolic co-morbidities in the developed countries has increased dramatically since the midtwentieth century ${ }^{39}$. Sedentary lifestyles and increased food consumption in combination with a widespread polygenetic susceptibility are considered to be the major causes for the obesity epidemic ${ }^{40}$, which is potentially further aggravated by the widespread use of antibiotics ${ }^{29}$. In addition, evidence is accumulating for a role of the gut microbiota in mediating some of the environmental effects in obesity pathogenesis. Following the discovery in 2006 that a transferrable obesity-associated microbiota can induce weight gain in lean mice ${ }^{41}$, subsequent epidemiological studies have shown differences in the gut microbiota of individuals with obesity and lean individuals. At the species level, twin studies have shown that the abundance of SCFA producers such as Eubacterium ventriosum and Roseburia intestinalis is associated with obesity ${ }^{42}$, whereas butyrate producers such as Oscillospira spp..$^{43}$ and the methanogenic archaeon Methanobrevibacter smithii may associate with leanness ${ }^{44}$. Another metagenome-wide association study of lean individuals and individuals with obesity showed that the abundance of Bacteroides thetaiotaomicron, a glutamate-fermenting commensal, was markedly decreased in individuals with obesity and was inversely correlated with serum glutamate concentration ${ }^{45}$. Furthermore, gavage of mice with $B$. thetaiotaomicron protected against adiposity, pointing to possible future modalities for obesity intervention targeting the gut microbiota with potential probiotic or microbial compounds (BOX 3). Analyses of gut microbial pathways and gene families suggest that obesity is associated with a decreased capacity for unidirectional conjugation, which transfers genetic material between bacteria, and a reduction in superoxide reductase, potentially leading to intestinal oxidative stress ${ }^{46}$ (FIG. 2). Observational studies have been complemented by a faecal microbiota transplantation study demonstrating that transplantation of stools from twins discordant for obesity into germ-free mice transfers, in a diet-dependent manner, the phenotype of the human donor to the recipient animal ${ }^{47}$. This landmark study provides the rationale for the ongoing intense search for gut microbial messengers linking the gut microbiota with whole-body energy metabolism. In studies of the potential impact of altered gut microbiota in the development of chronic disorders including obesity, however, it is important to bear in mind that various drugs may influence the gut microbiota. For instance, intake of statins, which are cholesterol-lowering drugs widely prescribed for the prevention of arteriosclerosis, has been linked with a health-promoting gut 


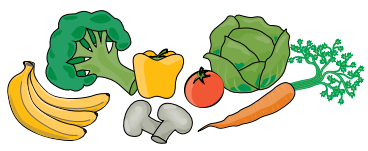

Diet low in animal fat and protein but high in plant fibres

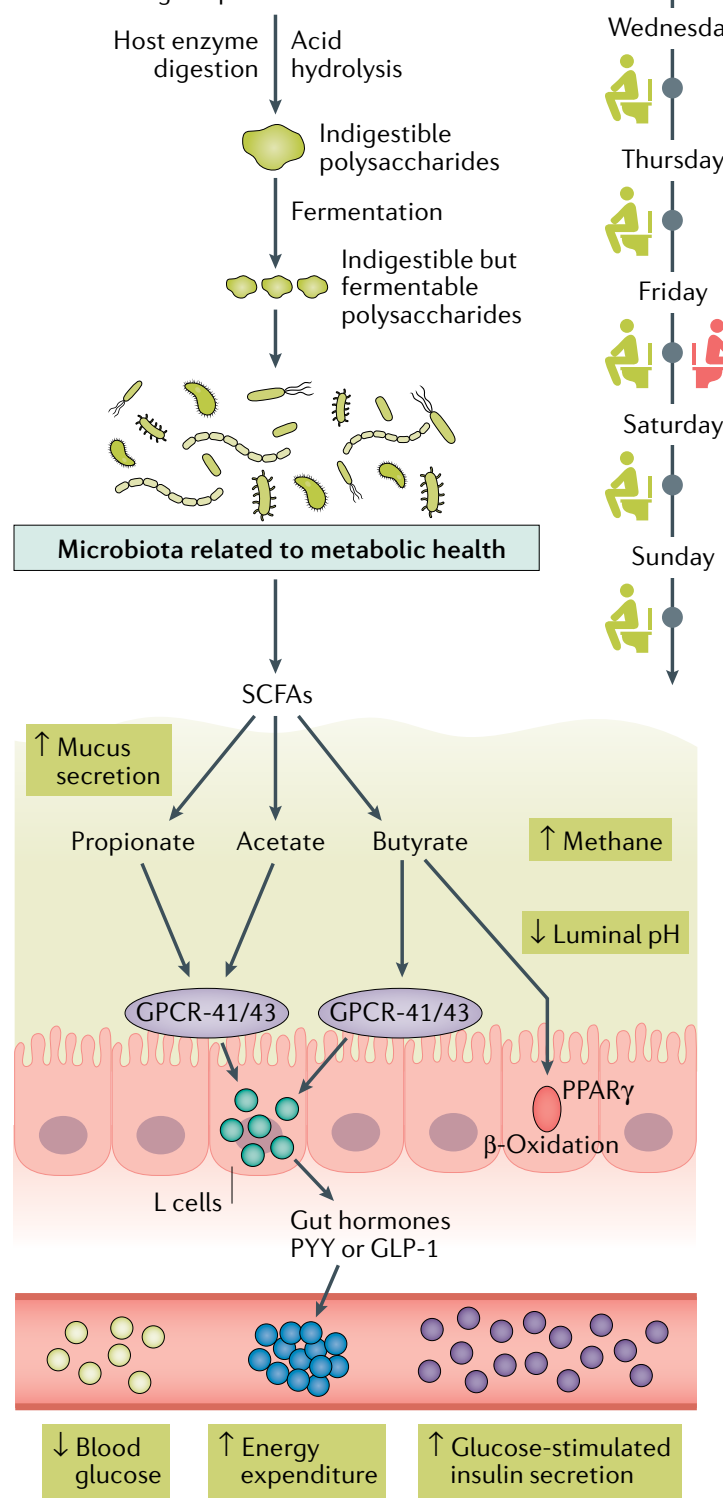

microbiota $^{48}$. Obviously, observations of any effects of microbiota-modifying drugs ideally need to be followed up in interventional studies to address any causality of the relationships.

Type 2 diabetes and prediabetes link with altered gut microbiota. Indirect evidence that the intestinal microbiota together with the hormonal secretion capacities of the distal gut may be involved in glucose regulation comes from large-scale epidemiological studies showing that, compared with individuals without colectomy, patients with total colectomy have an increased risk of $\mathrm{T} 2 \mathrm{D}^{49}$. In addition, mechanistic studies in rodents

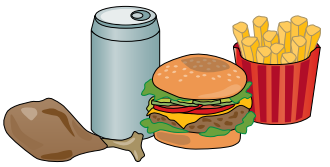

Diet high in animal fat and protein but low in plant fibres

$$
\begin{array}{r|r}
\text { Host } \\
\text { proteases }
\end{array} \text { Proteolysis }
$$

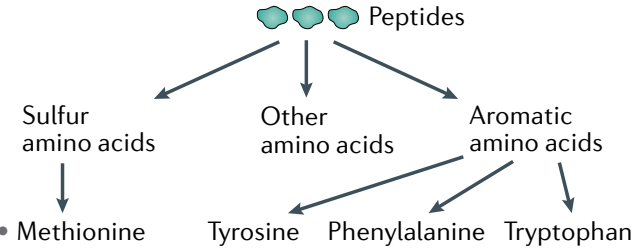

- Cystine

- Cysteine

- Taurine

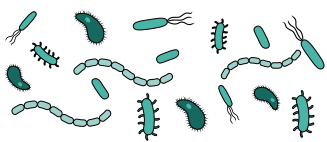

Aberrant microbiota related to metabolic diseases
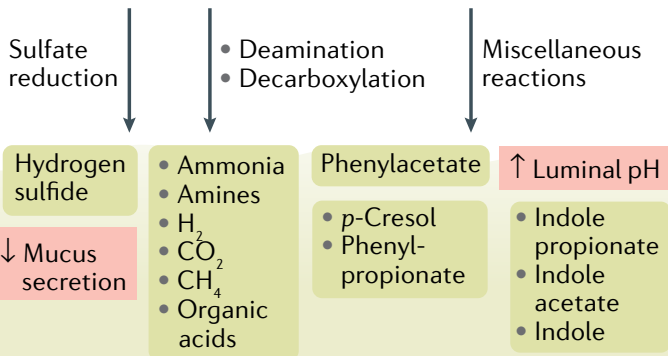

$\uparrow$ Acetaldehyde

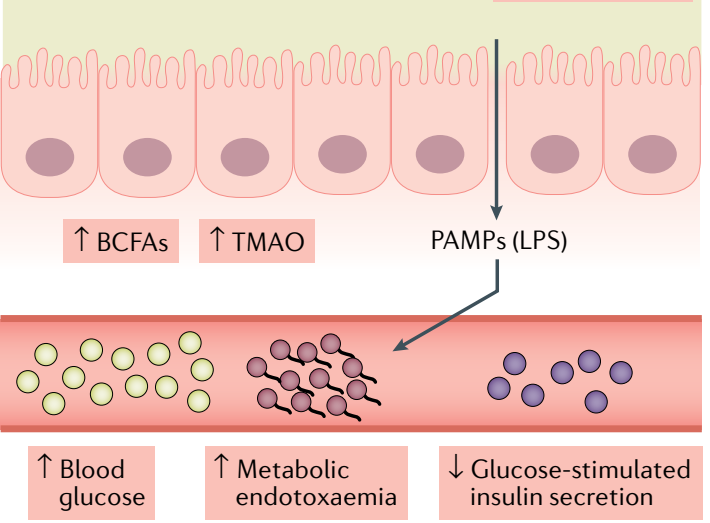

have shown that hyperglycaemia may increase intestinal barrier permeability through a GLUT2-dependent transcriptional reprogramming of intestinal epithelial cells and alteration of tight junction integrity, subsequently causing a leaky mucosa $\mathrm{a}^{50}$. Therefore, there is major interest in understanding whether an aberrant gut microbiota is involved in triggering or sustaining elevated blood glucose in T2D and its precursor states. T2D represents $\sim 90 \%$ of all diabetes cases ${ }^{51}$ and, like obesity, is growing in incidence and prevalence, affecting between 5 and $15 \%$ of the adult population, making it the most common endocrine disorder ${ }^{52}$. The aetiology of T2D involves a combination of hundreds of genetic 
Fig. 1 Impact of diet on gut microbiota and host metabolism. The balance between metabolically healthy microbiota and dysbiosis is crucial. Homeostasis is maintained by various factors, such as host genetics, diet, daily number of defecations, physical activity, smoking and drug usage. a |A metabolically healthy microbiota (mainly achieved by a high-fibre, low animal fat and low animal protein diet) is illustrated. The indigestible but fermentable polysaccharides are metabolized by the microbiota of the large intestine and are fermented to produce an array of compounds, and to stimulate a thick intestinal mucus layer and strong barrier functions. Microbial production of short-chain fatty acids (SCFAs) provides an additional energy source for colonocytes and causes a decrease in luminal $\mathrm{pH}$. The SCFAs acetate, butyrate and propionate can bind to $\mathrm{G}$ protein-coupled receptor (GPCR)-41 and GPCR-43, which are expressed on enteroendocrine $L$ cells, and subsequently induce secretion of glucagon-like peptide 1 (GLP-1) and peptide YY (PYY) that contribute to increased energy expenditure ${ }^{162}$, reduced food intake ${ }^{163}$ and improved glucose metabolism and insulin secretion ${ }^{164}$. Butyrate is an activator of the peroxisome proliferator-activated receptor- $\gamma$ (PPAR $\gamma$ ) and a stimulator for $\beta$-oxidation and oxygen consumption in the gut, which maintains an anaerobic environment in the gut lumen ${ }^{114}$. b | Microbial dysbiosis induced by a high animal fat and protein diet, sedentary life, smoking, alcohol intake and relatively infrequent defecation may result in a leaky mucosa, intestinal and systemic inflammation and reduced production of SCFAs, leading to less gut hormones being secreted by $L$ cells. In the fermentation process, complex proteins are first cleaved by various bacterial peptidases, proteases and endopeptidases to release free amino acids and short peptides that then undergo fermentation ${ }^{165}$. Of note, a dysbiotic microbiota is often associated with a prolonged colonic transit time, resulting in a shift in colonic metabolism leading to increased microbial proteolysis, even though the preferred substrate for bacterial fermentation is fermentable dietary fibres and bacteria will not switch to protein metabolism until fermentable polysaccharides are depleted ${ }^{166}$.As a result of increased protein fermentation, branched-chain fatty acids (BCFAs; 2-methyl butyrate, isobutyrate and isovalerate), trimethylamine, organic acids, gases $\left(\mathrm{H}_{2} \mathrm{~S}\right.$ (malodorous), $\mathrm{H}_{2}$ and $\mathrm{CO}_{2}$ ) and trace amounts of phenols, amines, indoles and ammonia are produced, causing an increase in luminal $\mathrm{pH}^{167}$. Altogether, such changes in the microbial environment and metabolites cause a leakage of pathogen-associated molecular patterns (PAMPs) including lipopolysaccharides (LPS) that become increased in the blood and trigger systemic low-grade inflammation and insulin resistance ${ }^{147}$. It should be noted, however, that some indole derivatives such as 3-indolepropionic acid produced by fermentation of dietary fibres have been shown to improve glucose metabolism ${ }^{137}$. TMAO, trimethylamine $\mathrm{N}$-oxide.

variants and a series of environmental factors, which are shared with obesity ${ }^{53}$. Most individuals are either overweight or obese, and the pathophysiology in the early stages of these cases is characterized by insulin resistance of primarily skeletal muscles and liver and adipose tissues, and a compensatory increased biosynthesis and secretion of insulin. In the presence of continued insulin resistance, insulin biosynthesis declines, which is followed by an aggravation of hyperglycaemia ${ }^{5,55}$. Besides hyperglycaemia, the T2D phenotype often exhibits dyslipidaemia with elevated circulating levels of triglycerides and low-density lipoprotein cholesterol, hypertension and an increased tendency for platelet aggregation. All of these abnormalities are risk factors for premature $\operatorname{arteriosclerosis}^{55,56}$. Consequently, individuals with T2D are, in addition to health behaviour modifications, treated with multiple medications targeting both elevated plasma glucose and risk factors for cardiovascular co-morbidities. However, the multidrug regimens affect the gut microbiota of these individuals in many different ways ${ }^{57}$. Therefore, recent epidemiological studies attempting to elucidate links between the gut microbiota and T2D have focused on the drug-naive early stages of T2D, called prediabetes. Individuals with prediabetes have elevated plasma glucose values that are within the non-diabetes range but raised to a level where they are at an increased risk of developing overt T2D and ischaemic cardiovascular disease $\mathrm{e}^{11}$. In these drug-naive individuals with prediabetes, the gut microbiota exhibits a loss of butyrate-producing taxa, a decrease in abundance of Akkermansia muciniphila and an increase in abundance of bacteria with pro-inflammatory potentials ${ }^{11,58}$ (FIG. 2). Gestational diabetes mellitus (GDM), which may occur in the third trimester of pregnancy and is estimated to affect $7-10 \%$ of all pregnancies, is another prediabetic state $^{59}$. In GDM, diagnostic criteria for plasma glucose are set at lower thresholds than for other forms of diabetes owing to the toxic effects on fetus development of even slightly elevated glycaemia ${ }^{59}$. Interestingly, compared with pregnant women with normoglycaemia in the same trimester, women with GDM have a disrupted gut microbiota composition, and differences in the gut microbiota are still detectable between the two groups of women 8 months after pregnancy. Both during and after pregnancy, the gut microbiota of GDM resembles the aberrant microbiota composition reported in non-pregnant individuals with $\mathrm{T} 2 \mathrm{D}^{60}$, where a reduced abundance of butyrate-producing bacteria has been consistently found in addition to an often reduced bacterial richness and an increase in opportunistic pathogens ${ }^{8,61}$. At the functional potential level, the gut microbiome of individuals with T2D is enriched in multiple pathways. These include an enrichment of pathways involved in sugar-related membrane transport to increase cellular glucose uptake, branched-chain amino acid (BCAA) outward transport to induce insulin resistance, methane metabolism related to an anaerobic intestinal environment, xenobiotic degradation, metabolism to promote drug resistance and sulfate reduction to reduce insulin sensitivity $^{8}$ (FIG. 2). However, interpretation of gut microbiota profiles from individuals with T2D who are being treated with multiple drugs is problematic owing to drug confounders. Among the drugs that are frequently prescribed for T2D, the anti-hyperglycaemic drug metformin appears to change the gut microbiota most, with effects on the relative abundance of multiple genera and species, such as Escherichia and Intestinibacter, and enhancement of several microbiome functional potentials, such as propionate and butyrate production that induces intestinal gluconeogenesis ${ }^{62-64}$. In fact, the gut microbiota has been suggested to mediate some of the anti-hyperglycaemic effects of metformin through enhanced production of SCFAs and unconjugated bile acids, which activate intestinal gluconeogenesis, and an increase in intestinal gluconeogenesis resulting in a lowering of glycaemia ${ }^{65,66}$. However, any effects that metformin-induced changes of gut microbiota have on overall glucose regulation may be modest. Consistent with this notion, it has been reported that in germ-free or antibiotic-treated mice, the effect of metformin on measures of dysmetabolism remains largely unaffected ${ }^{67}$. Metformin treatment is also known to cause transient or persistent intestinal discomfort in around one-third of individuals, which in part may be attributed to increased gas production by some Escherichia spp. and an enrichment of various virulence factors ${ }^{63,64,68}$.

Altogether, these studies investigating the role of the gut microbiota in T2D highlight several important findings and shortcomings. The microbiome in individuals with overt $\mathrm{T} 2 \mathrm{D}$ and prediabetes appears to be 


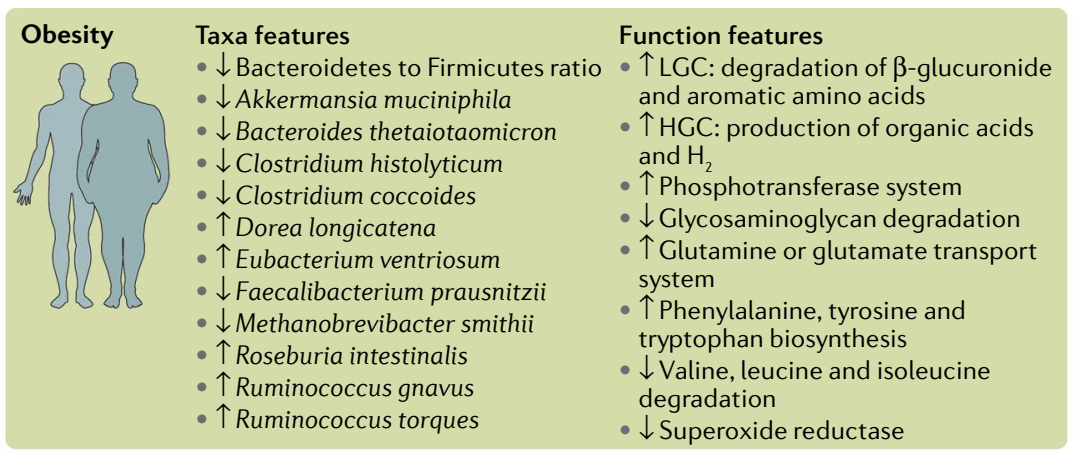

Malnutrition
Taxa features
- $\downarrow$ Anaerobes to facultative
anaerobes ratio
- $\downarrow$ Bifidobacterium longum
- $\downarrow$ Bifidobacterium pseudolongum
Function features
- $\downarrow$ Lantibiotics production
- $\downarrow$ Energy harvest
- $\downarrow$ Immune protection
- $\downarrow$ Vitamin biosynthesis
- $\uparrow$ Pathogenic factors

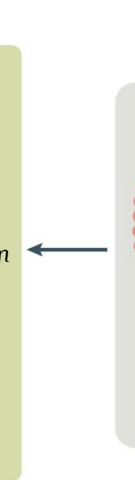

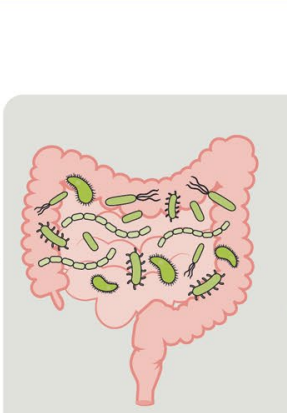

Healthy microbiota

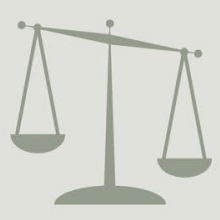

2

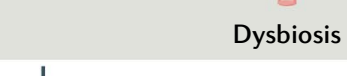

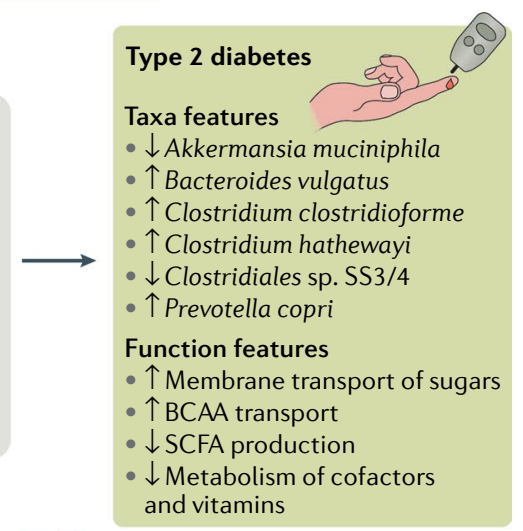

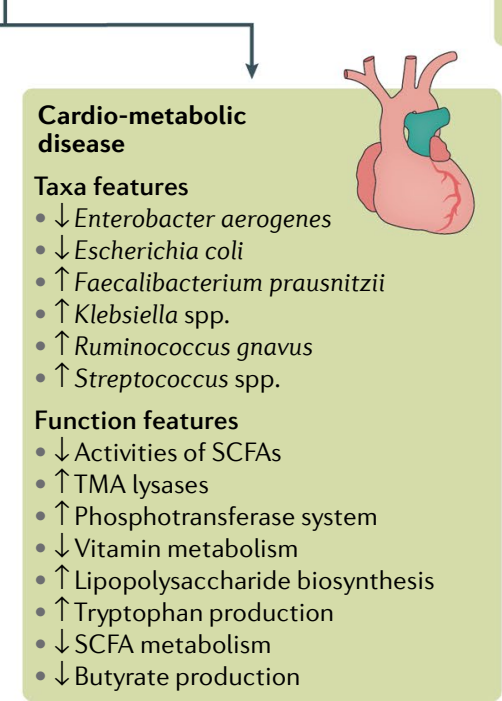

Fig. 2 | Some of the reported intestinal microbial taxonomic and functional features linked to common metabolic disorders. An overview of selected key gut microbiome features related to metabolic diseases. Multiple studies have implicated bacterial species and functional features in metabolic diseases; however, results differ between studies. The association results from metagenomic studies have been summarized. Selected microbial taxonomic features are listed in addition to their directions in metabolic diseases. The list is not comprehensive of all altered taxonomic or functional features but represents common patterns observed across studies. AAA, aromatic amino acid; BCAA, branched-chain amino acid; HGC, high gene count; LGC, low gene count; SCFA, short-chain fatty acid; TMA, trimethylamine; $\downarrow$, lower abundance in metabolic diseases when compared with control; $\uparrow$, higher levels in metabolic diseases when compared with control.

relatively depleted in bacterial butyrate producers and to exhibit an increase in species with a pro-inflammatory functional potential. However, none of these changes is specific to T2D but they occur in many chronic non-communicable disorders characterized by a clinically silent low-grade inflammation. So far, attempts to transfer gut microbiota from drug-naive individuals with T2D or prediabetes to germ-free mice to reproduce the $\mathrm{T} 2 \mathrm{D}$ phenotype have failed ${ }^{11}$.
Gut microbial dysbiosis linked to cardio-metabolic diseases. Arteriosclerosis is one of the leading causes of morbidity and mortality in the western world ${ }^{69}$. People who suffer from arteriosclerosis frequently have prior clinically silent metabolic dysfunctions for many years, including elevated circulating concentrations of glucose, insulin ${ }^{70}$ and lipids ${ }^{71}$ in addition to insulin resistance and low-grade inflammation ${ }^{72}$. Therefore, it is a major challenge to disentangle the possible impact of an 
Atherogenesis

The dynamic process of forming atheromas (also called plaques), that is, accumulated inflammatory cells, lipids, cell debris, minerals and connective tissue in and on the walls of an artery forming a swelling that narrows the arterial lumen and restricts the flow of blood.

Thrombosis

The formation of a blood clot, known as a thrombus, within a blood vessel. The blood clot that consists of platelets, red blood cells and fibrin proteins obstructs the flow of blood through the blood vessel.

Stroke

An acute brain insult where compromised blood flow in atherosclerotic arteries or bleeding from brain arteries causes damage to brain tissues often resulting in various paresis

Myocardial infarction (Also known as an acute heart attack or acute coronary syndrome). An event that occurs when blood flow is acutely compromised or is completely stopped to a part of the heart muscle (myocardium), causing severe damage to the heart.

\section{Atherosclerosis}

The build-up of cholesterol,

other lipids, inflammatory cells and calcium in artery walls,

which can restrict blood flow.

Atherothrombosis

The formation of a blood clot within an artery that is affected by arteriosclerosis.

Plaque instability Vulnerable arterial wall plague that intermittently ruptures giving rise to circulating plaque fragments called emboli, which may cause myocardial infarction or stroke.

Intima

The innermost coating of the vessel wall including the endothelial surface at the lumen.

Steatosis

An abnormal retention of lipids within an organ. The term is most often used about a fatty liver. aberrant gut microbiota on early-stage metabolic perturbations from an imbalanced gut microbiota that, at a stage distal to the dysmetabolic stage, is escalating the pathogenesis of ischaemic heart disease. On top of this, individuals with CMD are, as in T2D, heavily medicated, making it challenging to tease apart authentic arteriosclerosis signals in the gut microbiota from signatures induced by complex medication and pre-morbidities and co-morbidities. So far, these challenges have not been systematically addressed. A metagenome-wide association study of faecal samples from individuals with CMD reported that the gut microbiome of these individuals, without adjustments for pre-morbidities or co-morbidities, exhibits enriched abundances of Enterobacteriaceae, including Escherichia coli, Klebsiella spp. and Enterobacter aerogenes, as well as an increased abundance of oral cavity species when compared with healthy control subjects ${ }^{73}$ (FIG. 2). By contrast, individuals with CMD had a reduced abundance of Bacteroides spp. and anti-inflammatory Faecalibacterium prausnitzi ${ }^{74}$. At the functional level, a decrease in some fermentation capacities was seen but an enrichment in the phosphotransferase system, amino acid transporters and enzymes involved in the synthesis of LPS and trimethylamine (TMA) ${ }^{73}$ was also seen. Recently, it was shown that ischaemic heart failure is associated with a dysbiotic gut microbiota with an increased abundance of Ruminococcus, Acinetobacter and Veillonella spp. and a decreased abundance of Alistipes, Faecalibacterium and Oscillibacter spp. ${ }^{75}$. At the functional level, the microbiome of these patients was enriched in genes involved in the biosynthesis of LPS and trimethylamine $\mathrm{N}$-oxide $(\mathrm{TMAO})^{75}$. Taken together, these studies of CMD point to a more inflammatory and a less fermentative microbiome, even though it remains to be determined which components of the gut microbiota are involved in earlystage dysmetabolism and which are related to a more distal atherogenesis stage.

Of special interest is the discovery of the TMAO pathway with the involvement of the gut microbiota and relations to ischaemic vascular diseases ${ }^{76}$. TMA is synthesized by gut microbiota from dietary phosphatidylcholine, lecithin and L-carnitine, which are found not only in meat from four-legged animals but also in poultry, seafood and eggs. TMA enters the portal circulation and is oxidized to TMAO in the liver. Experiments in rodents demonstrate that feeding dietary TMAO or its dietary precursors causes an acceleration of arteriosclerosis, induces platelet aggregation and enhances the thrombosis potential ${ }^{77}$. Moreover, inhibition of intestinal bacterial TMA production with 3,3-dimethyl-1-butanol (a TMA-lyase inhibitor) attenuates arteriosclerosis and thrombosis ${ }^{78}$. Epidemiological studies showing positive relationships between the plasma concentrations of TMAO and an increased prevalence of stroke and myocardial infarction as well as an increase in premature mortality of people with stable coronary artery ischaemia lend support to the outcome of the mechanistic experiments that TMAO is a biomarker of $\operatorname{arteriosclerosis}^{79,80}$.

Still, there are inconsistencies in available results. For instance, mice fed a diet enriched in L-carnitine have increased plasma TMAO concentrations but unexpectedly reduced aortic lesion sizes without changes in plasma lipids, thereby indicating a potential beneficial role for L-carnitine in the prevention of atherosclerosis independently of an increase in plasma TMAO, in this specific mouse model ${ }^{81}$. Moreover, there are numerous foods that are rich in TMAO itself, such as some cold water-dwelling fish, the intake of which is generally considered beneficial for cardiovascular health ${ }^{82}$. Lately, the contradictory results may have been reconciled to some extent $^{83}$. Recent animal and human studies suggest that the influence of TMAO on vascular biology is dependent on dietary-microbiome interactions and host genetics, which may explain some of the apparent controversies of TMAO's involvement in atherothrombosis. In rodent experiments, it was found that TMAO can be directly produced in the gut in addition to oxidation of TMA in the liver, and blood TMAO levels increased after feeding the animals with a high-fibre diet or a high-choline diet. In two different genetically modified mouse models that are predisposed to arteriosclerosis, investigators could not find evidence of any relationships between TMAO generation and accelerated atherogenesis ${ }^{83}$. However, in the atherosclerosis-prone animals, circulating TMAO concentrations linked directly with features of plaque instability, including biomarkers of platelet aggregation, intra-plaque bleeding and inflammation. In a population-based observational study of adults, the same authors could not find evidence of correlations between plasma TMAO levels and markers of arteriosclerosis such as intima thickness of carotid arteries or calcium scores of coronary arteries ${ }^{83,84}$. Thus, it appears that a major role of the partially gut microbiota-derived TMAO, in relation to ischaemic vascular disorders, may be an aggravation of atherogenesis in individuals with already established ischaemic heart disease or stroke through mechanisms primarily involving plaque instability.

Dysbiosis of the intestinal microbiota in metabolic liver diseases. Non-alcoholic fatty liver disease (NAFLD), frequently regarded as the hepatic manifestation of the metabolic syndrome, occurs in many countries with a prevalence of $20-40 \%$ of the adult population ${ }^{3}$. NAFLD comprises a wide spectrum of diseases ranging from simple steatosis to non-alcoholic steatohepatitis (NASH), which is the inflammatory, aggressive form of NAFLD $^{85}$. There is growing recognition that a disrupted gut microbiota may be one of several crucial factors in the pathophysiology of NAFLD and NASH. Individuals with NAFLD have an increased abundance of species assigned to Clostridium, Anaerobacter, Streptococcus, Escherichia and Lactobacillus, whereas Oscillibacter, Flavonifaractor, Odoribacter and Alistipes spp. are less abundant ${ }^{86}$. In comparison, the abundance of Proteobacteria, Enterobacteriaceae and Escherichia spp. is elevated in patients with $\mathrm{NASH}^{87}$. Concordantly, children with steatosis or NASH are depleted in Oscillospira spp. accompanied by higher abundance of Dorea and Ruminococcus spp. when compared with controls $^{88}$. The microbiota alterations are associated with higher faecal concentrations of 2-butanone and 
Portal endotoxaemia

Endotoxins, primarily bacterial lipopolysaccharides, which are absorbed from the intestines into mensenteric and liver veins (portal drainage)

Liver cirrhosis

A chronic liver disease caused, for instance, by alcohol abuse or virus infection with impairment of multiple liver functions owing to replacement of normal liver tissues by scar tissue.

Hepatic encephalopathy A spectrum of cognitive and neuro-psychiatric

abnormalities such as personality changes, anxiety,

confusion, fatigue, shaky hands or seizures caused by severely impaired liver function.

Holobiont

The unique and discrete collective of a macro-organism - a host - and the complex microbial communities for which the macro-organism is the habitat.

Anorexigenic hormones These appetite-decreasing hormones include

glucagon-like peptide 1 (GLP-1) and peptide YY (PYY) both produced by specialized intestinal cells and leptin produced by adipocytes and intestinal cells.

Leptin

A hormone predominantly synthesized in adipose cells and enterocytes in the small intestine that helps to regulate energy balance by inhibiting hunger, which in turn diminishes fat storage in adipocytes. 4-methyl-2-pentanone that cause hepatocellular toxicity in individuals with metabolic liver diseases compared with healthy controls ${ }^{88}$.

Additionally, given that the microbiota associated with NALFD is enriched in ethanol-producing bacteria such as E. coli, it has been hypothesized that the aberrant gut microbiome of individuals with NAFLD produce more ethanol than microbiomes of healthy individuals, as evidenced by increased concentrations of intrinsically generated ethanol in the circulation and breath $^{87,89}$ (FIG. 2). Ethanol activates nuclear factor- $\kappa B$ $(\mathrm{NF}-\kappa \mathrm{B})$ signalling pathways and causes tissue damage by impairing gut barrier function and contributing to portal endotoxaemia ${ }^{90,91}$. In the liver of individuals with NALFD, the detoxification pathway is weakened, providing a constant source of reactive oxygen species that potentially cause oxidative damage to the hepatocytes, which in turn may induce hepatic inflammation and steatohepatitis $^{92}$.

Similarly, individuals with liver cirrhosis have a substantially altered microbiota, including an enrichment of Proteobacteria and Fusobacteria phyla ${ }^{9}$. The functional potentials of the gut microbiome in individuals with liver cirrhosis and liver failure are massively altered compared with healthy individuals. The changes include significant enrichment of both assimilation and dissimilation of nitrate to or from ammonia as signatures of hepatic encephalopathy. An increase in denitrification capacity may relate to the higher nitrogen production, whereas increased $\gamma$-aminobutyric acid (GABA) biosynthesis and GABA shunt functions relate to hydrodynamic venous shunting in liver failure. An increase of microbial haem biosynthesis may contribute to liver damage because of protoporphyrin toxicity, whereas an increase in phosphotransferase systems may indicate an increased demand for protein following liver tissue damage $^{9,93}$

Apart from the above-mentioned observations, indicating links between gut microbial composition and function and metabolic liver disorders, human and animal faecal transplantation studies show that a high abundance of the alcohol-producing bacterial species Klebsiella pneumoniae in the gut accelerates NAFLD pathogenesis ${ }^{94,95}$. Additionally, an integrative multi-omics approach in women with obsesity without diabetes identified phenylacetate as a microbial metabolite contributing to the accumulation of lipids in the liver and, hence, to $\mathrm{NASH}^{96}$.

Disrupted gut microbiota in malnutrition. Globally, malnutrition affects $\sim 160$ million people and is the leading cause of death in children under the age of 5 years ${ }^{97,98}$. Breastfeeding, food and water security are major protective factors against malnutrition and are crucial factors in the maturation of the healthy gut microbiota, characterized by a transient bifidobacterial bloom before a rise in anaerobes during late infancy ${ }^{99}$. Early loss of Bifidobacterium longum and Bifidobacterium pseudolongum, two key members in mother milk, represents some of the first disruptions in the intestinal dysbiosis of severe acute malnutrition (SAM) ${ }^{100}$ (FIG. 2). B. longum and $B$. pseudolongum appear to be crucial in shaping the early gut microbiota in infants, partly through secretion of broad-spectrum lantibiotics ${ }^{101}$. Following this, SAM dysbiosis is mainly accompanied by an aberrant inversion of the ratio of anaerobes to facultative anaerobes. This inversion might be corrected under appropriate dietary treatment, eventually leading to microbial communities enriched in anaerobes ${ }^{102}$. However, in cases where malnutrition continues, the loss of the healthy mature anaerobic gut microbiota gradually leads to a deficiency in energy harvest ${ }^{41}$, immune responses ${ }^{103}$ and vitamin synthesis ${ }^{104}$, and links with chronic malabsorption, diarrhoea and systemic invasion from pathogenic bacteria ${ }^{105}$. Current dietary therapeutic regimens for SAM are not based on knowledge about the developmental biology of the intestinal microbiota. However, a recent study designed diets using pig and mouse models to nudge the microbiota into a mature post-weaning state, which could entrain maturation of the microbiota of children with SAM and restore their metabolic and growth profiles to a healthier trajectory ${ }^{106}$. Such dietary regimens urgently need to be tested in long-term randomized clinical trials to evaluate the effects of maturating the gut microbiota on weight gain and overall physiology of affected children.

\section{Bacterial messengers affect metabolism}

An overwhelming number of reports demonstrate that disruptions in gut microbiome taxonomy and functional potential relate to numerous pathological phenotypes. The majority of studies in humans and animals are observational and lack experimental mechanistic data. However, as revealed by the genetic repertoire of the human gut microbiome, the trillions of commensal or mutualistic bacteria and archaea are an immense chemical factory that can synthesize a multitude of compounds needed for their own existence and survival with their host, but also compounds that affect the entire holobiont. In the following sections, we selectively review some reported intestinal microbial products that affect host energy homeostasis, body adiposity, glucose tolerance, insulin sensitivity, inflammation and endocrine regulation (FIG. 3).

Microbiota affecting energy homeostasis and body adiposity. For their own energy supply, the gut microbiota ferment energy-yielding nutrients, especially complex carbohydrates and, to lesser extent, proteins, monosaccharides, SCFAs and amino acids. However, the microbiota produces large amounts of fermented nutrients that benefit their host, corresponding to 5-10\% of the daily energy needs of a human ${ }^{107}$. Here, we first focus on the SCFAs butyrate, propionate and acetate, which affect energy metabolism and body adiposity in various ways in addition to being simple substrates for overall host energy turnover and nutrients for colonic cell metabolism. Both propionate and butyrate are predominantly anti-obesogenic through their stimulation of anorexigenic hormones and leptin synthesis ${ }^{108,109}$. Mice treated with a butyrate precursor drug (tributyrin) are protected from diet-induced obesity, insulin resistance and hepatic steatosis ${ }^{110}$. Butyrate also suppresses inflammation in various tissues, enhances the differentiation of 
colonic anti-inflammatory regulatory $\mathrm{T}$ cells and induces the NOD, LRR and pyrin domain-containing protein 3 (NLRP3) inflammasome in a G protein-coupled receptor manner ${ }^{111,112}$. Another $G$ protein-coupled receptor, the olfactory receptor 78 , which may modulate blood pressure regulation, can also be directly activated by acetate and propionate ${ }^{113}$. Additionally, increased butyrate production activates the peroxisome proliferator-activated receptor- $\gamma$ (PPAR $\gamma$ ), leading to $\beta$-oxidation and oxygen consumption in the gut, a phenomenon that contributes to the maintenance of anaerobic conditions in the gut lumen $^{114}$ (FIG. 1).

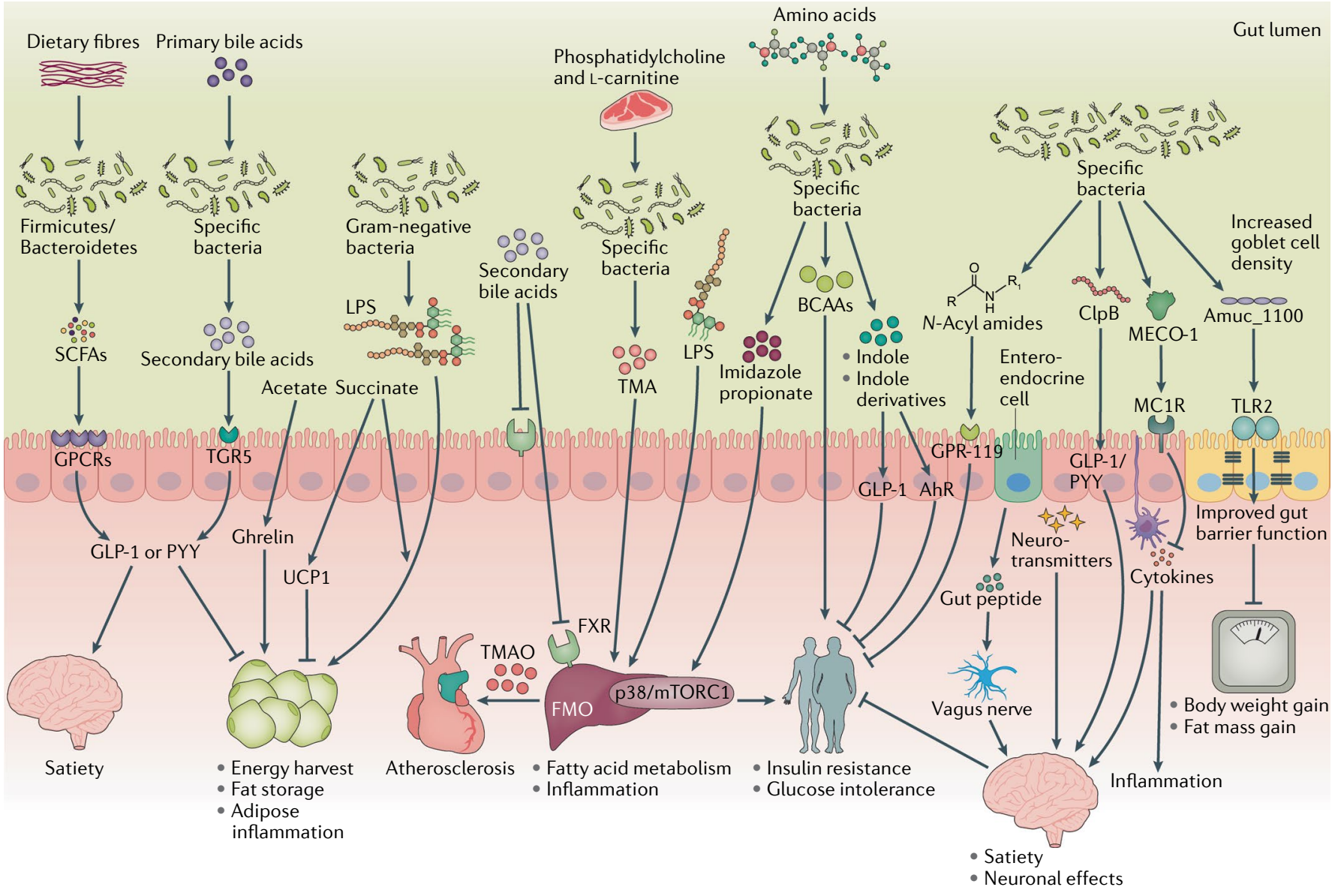

Fig. 3 | Microbial messengers regulate host metabolism. An overview of some of the intestinal microbial compounds affecting host energy homeostasis, body adiposity, inflammation, glucose regulation, insulin sensitivity and hormone secretion. Fermentation of the dietary fibre by gut Firmicutes and Bacteroidetes produces the short-chain fatty acids (SCFAs) butyrate, propionate and acetate, which influence host metabolism in multiple ways by acting on G protein-coupled receptors (GPCRs) expressed by enteroendocrine cells. Acetate and butyrate stimulates glucagon-like peptide 1 (GLP-1) and peptide YY (PYY) release with effects on the pancreas (GLP-1-induced insulin biosynthesis) and on the brain (PYY-induced satiety), and acetate may enhance fat storage by inducing secretion of ghrelin. Microbial-derived succinate drives expression of uncoupling protein 1 (UCP1), thereby increasing thermogenesis in adipose tissue. However, succinate has pro-inflammatory properties on lipopolysaccharide-activated macrophages and may thus contribute to adipose tissue inflammation and insulin resistance. Lipopolysaccharides (LPS) are pro-inflammatory compounds derived from Gram-negative bacterial membranes that promote inflammation. Primary bile acids are converted by gut microbiota to secondary bile acids that act through the TGR5 receptor to promote GLP-1 release enhancing thermogenesis in adipose tissue. Gut microbiota produces trimethylamine (TMA) by metabolizing dietary phosphatidylcholine and L-carnitine. TMA is $\mathrm{N}$-oxidized in the liver by flavincontaining monooxygenase (FMO) to trimethylamine $\mathrm{N}$-oxide (TMAO), which contributes to atherosclerosis. Imidazole propionate, a bacterial metabolite derived from histidine, contributes to insulin resistance. Gut bacterial-derived branched-chain amino acids (BCAAs) correlate, in cases of high fat intake, with insulin resistance in both humans and rodents. Other bacterial metabolites such as indole and its derivatives bind to the aryl hydrocarbon receptor (AhR). Indolepropionic acid is linked with improved insulin secretion and insulin sensitivity and a decreased risk of type 2 diabetes. Gut bacteria produce $\mathrm{N}$-acyl amide, an endocannabinoid mimic that regulates host glucose metabolism by binding to the $G$ protein-coupled receptor 119 (GPR119). Proteins secreted by gut bacteria also modulate paracrine or endocrine action. $\mathrm{ClpB}$, a protein secreted by Escherichia coli, is involved in regulation of appetite. Melanocortin-like peptide of E. coli (MECO-1), which is a structural and functional analogue to a-melanocytestimulating hormone and adrenocorticotropin, acts via the mammalian melanocortin-1 receptor (MC1R), inhibiting cytokine release in response to pro-inflammatory stimuli. In the large intestine, pro-inflammatory changes involve increased production of cytokines such as IL-1 $\beta$ and IL-18, and reduced IL-10 and IL-22. Amuc_1100, expressed on the outer membrane of Akkermansia muciniphila, improves gut barrier function with increased goblet cell density through Toll-like receptor 2 (TLR2) and partially recapitulates the beneficial effect of the live A. muciniphila bacterium on energy metabolism and insulin sensitivity. Host metabolism is also influenced by gut bacterially synthesized neurotransmitters (that is, catecholamine, histamine, $\gamma$-aminobutyric acid and serotonin) or gaseous neurotransmitters $\left(\mathrm{NO}\right.$ and $\mathrm{H}_{2} \mathrm{~S}$ ). Arrow represents stimulation, arrow with bar indicates inhibition. FXR, farnesoid X receptor; mTORC1, mechanistic target of rapamycin complex 1. 
In two independent studies, structural variations in the Anaerostipes hadrus genome that encode metabolic modules for inositol catabolism and for metabolizing 3-hydroxybutanoyl-CoA to butyrate show inverse relationships with body weight, waist circumference and body mass index, potentially pointing to mechanistic links between these metabolic pathways and host metabolic health ${ }^{17}$.

A mouse study demonstrated that acetate, butyrate and propionate may have anabolic effects on host metabolism ${ }^{115}$, whereas another study demonstrated that long-term administration of an inulin-propionate ester significantly reduced weight gain, intra-abdominal adipose tissue distribution, intra-hepatocellular lipid content and insulin resistance in overweight adults ${ }^{116}$. Nevertheless, other investigators have reported conflicting findings for effects of propionate on host metabolism. A host genetics-driven increase in gut microbial production of butyrate improved the insulin response after an oral glucose tolerance test, whereas propionate causally increased the risk of $\mathrm{T} 2 \mathrm{D}^{117}$. In line with the latter finding, another study in mice suggested that propionate impairs insulin signalling through stimulation of the production of glucagon and fatty acid-binding protein $4(\mathrm{FABP} 4)^{118}$. Intake of a propionate-containing meal in humans resulted in a postprandial increase in plasma concentrations of glucagon, FABP4 and norepinephrine, leading to insulin resistance and compensatory hyperinsulinemia ${ }^{118}$. Finally, findings in a human pilot study suggested that glucose metabolism is more responsive to oral butyrate supplementation in lean individuals than in people with the metabolic syndrome, which argues against oral butyrate supplementation as a potential therapeutic remedy to improve glucose regulation in individuals with dysmetabolism ${ }^{119}$. These conflicting experimental results call for human studies in which the physiological effects of each SCFA are tested separately as well as in combinations, relative to their proportional concentrations in circulation, in order to properly and systematically investigate their net effects on glucose homeostasis, insulin secretion and insulin sensitivity.

Unlike butyrate and propionate, acetate may predominantly have obesogenic properties because it acts as a substrate for hepatic and adipocyte lipogenesis ${ }^{120}$. In mice, increased acetate production induced by an altered gut microbiota promotes hyperphagia through increased ghrelin secretion and enhances fat storage by increasing glucose-stimulated insulin secretion ${ }^{121}$. In humans, plasma concentrations of the intestinal hormones, peptide YY (PYY) and glucagon-like peptide 1 (GLP-1) are reported to increase following rectal or intravenous administration of acetate ${ }^{122}$.

Host energy homeostasis and body adiposity are also influenced by bile acids, and the gut microbiota converts primary bile acids to various secondary bile acids $^{123}$. Both categories of bile acid exert their metabolic functions by binding to cellular receptors in various organs of the body, including nuclear farnesoid X receptor (FXR) and TGR5, a G protein-coupled receptor ${ }^{124}$. Signalling via these receptors is implicated in glucose metabolism, yet in opposite directions; FXR impairs ${ }^{125}$ whereas TGR 5 promotes ${ }^{126}$ carbohydrate metabolism.
One of the primary bile acids, chenodeoxycholic acid, is a highly efficacious FXR ligand ${ }^{127}$, whereas the secondary bile acids lithocholic acid (LCA) and taurolithocholic acid, which in rodents are transformed from primary bile acids by intestinal bacteria ${ }^{123}$, are the two most potent endogenous TGR5 ligands ${ }^{128}$. TGR5 signalling in enteroendocrine L cells induces secretion of GLP-1, thereby improving liver function and enhancing glucose tolerance in obese mice ${ }^{126}$. LCA, a high-affinity TGR5 agonist, stimulates thermogenesis through browning of both white and brown adipose tissues ${ }^{129}$, whereas deoxycholic acid (DCA) and LCA have been suggested as partial antagonists of $\mathrm{FXR}^{130}$. Recently, a subgroup of secondary bile acids, C-6-hydroxylated secondary bile acids, have attracted attention as these bile acids may be associated with leanness in rodents ${ }^{131}$ and early weight loss in people with obesity following sleeve gastrectomy ${ }^{132}$.

Studies of the intestinal microbiome in several chronic metabolic disorders show increased functional potentials for metabolism of amino acids ${ }^{133}$. Whether these observations reflect primary disease-linked mechanisms or secondary phenomena is unclear. However, in the case of microbial break down of tryptophan, both in vitro and in vivo studies suggest that indole, a tryptophan microbial catabolite (FIG. 1), improves intestinal epithelial barrier functions ${ }^{134,135}$. Indole is also a signalling molecule involved in enhancing the release of the gut hormone GLP-1 from enteroendocrine L cells ${ }^{136}$, thereby indirectly influencing insulin secretion and appetite regulation. Similarly, the circulating concentration of 3 -indolepropionic acid (IPA), a catabolite involved in the tryptophan metabolic pathway, is in epidemiological studies reported to associate with improved insulin secretion and insulin sensitivity, and a lower likelihood of developing $\mathrm{T} 2 \mathrm{D}^{137}$.

Microbiota affecting insulin sensitivity and endocrine regulation. Elevated circulating concentrations of the BCAAs leucine, isoleucine and valine are a strong biomarker for insulin resistance and an increased risk of $\mathrm{T}_{2} \mathrm{D}^{138}$. In individuals with normoglycaemia who are insulin resistant, the gut microbiome has an increased potential to synthesize BCAAs, which is largely driven by an increased abundance of Prevotella copri and Bacteroides vulgatus, but a reduced potential for microbial BCAA uptake and BCAA catabolism primarily driven by Butyrivibrio crossotus and Eubacterium siraeum $^{14}$. These findings, supported by experiments in mice, suggest that the gut microbiome may contribute to increased plasma BCAA concentrations and insulin resistance under conditions of unhealthy dieting ${ }^{138}$. In heart failure, the catabolism of BCAAs is impaired and the accumulated BCAAs induce oxidative stress and disrupt mitochondrial function ${ }^{139}$.

Imidazole propionate is yet another microbial compound that contextually impairs insulin signalling ${ }^{140}$. In portal and peripheral blood of individuals with T2D, imidazole propionate was present at higher concentrations than in healthy individuals. Gut bacterial species such as Streptococcus mutans and Eggerthella lenta were verified as producers of imidazole propionate. In mice experiments, imidazole propionate worsened glucose 
tolerance and insulin signalling through activation of the mechanistic target of rapamycin complex 1 (mTORC1) pathway. In line with these findings, overexpression of the mTORC1-mediated insulin signalling pathway was found in liver tissue isolated from individuals with T2D ${ }^{140}$.

Both the host and the intestinal microbiome produce succinate, which may have multiple roles as a metabolic intermediate. One role relates to insulin sensitivity and inflammation, with effects that are also apparently contextual. In wild-type mice, microbial-derived succinate not only prevented obesity but also improved insulin sensitivity and glucose tolerance $\mathrm{e}^{141,142}$. By contrast, succinate might act as a metabolite in innate immune signalling. Thus, in LPS-activated macrophages, succinate stabilized the transcription factor hypoxia-inducible factor $1 \alpha$, which increased IL- $1 \beta$ production and triggered inflammation. Therefore, succinate may under some conditions contribute to insulin resistance that is triggered by inflammation ${ }^{143}$. Unexpectedly, pharmacologically raised circulating succinate concentrations in mice have been shown to be a driver for uncoupling protein 1 (UCP1)-dependent thermogenesis in brown adipose tissue, thereby boosting protection against diet-induced obesity and improving glucose tolerance ${ }^{142}$. Nevertheless, outcomes of studies of the impact of the gut microbiome on regulation of UCP1 in various mouse models remain inconsistent ${ }^{144,145}$. Whether microbial-derived succinate is relevant to comparable processes in human metabolism is unknown.

Bacterial LPS are the major outer surface membrane components present in most Gram-negative bacteria ${ }^{146}$. LPS are released at bacterial cell death and become strong stimulators of host immunity. When in excess, for instance owing to habitual intake of diets high in animal fat or in an extreme state such as sepsis-elicited endotoxaemia, LPS trigger local and systemic inflammation and insulin resistance ${ }^{147}$. Generally, several common chronic disorders that appear to be characterized by a relative disruption of the gut barrier, which elicits multiple imbalances in both intestinal and systemic immune competences, converge to cause insulin resistance, eventually leading to metabolic syndrome ${ }^{148,149}$.

Above, we have discussed how, for instance, SCFAs and indole interfere with endocrine regulation, but there are more examples of how gut bacterial compounds interact with hormone secretion or act as ligands for known hormone receptors. Several proteins secreted by $E$. coli, including $\mathrm{ClpB}$, which mimics the host peptide $\alpha$-melanocyte-stimulating hormone $(\alpha-M S H)$, affect food intake ${ }^{150}$ and meal patterns in mice $^{151}$. Mechanistically, the $\mathrm{ClpB}$ protein stimulates release of intestinal GLP-1 and PYY and activates brain anorexigenic pathways inducing satiety. In humans, higher plasma ClpB levels have been reported in individuals with eating disorders such as anorexia nervosa compared with circulating $\mathrm{ClpB}$ levels in healthy individuals ${ }^{152}$. Furthermore, some gut commensal bacteria harbour $\mathrm{N}$-acyl amide synthase genes, and their encoded lipids mimic binding of classical eukaryotic signalling molecules to $\mathrm{G}$ protein-coupled receptors ${ }^{153}$. In experiments where mice were fed an engineered bacterium that produces the lipid $\mathrm{N}$-acyl serinol, which is structurally similar to a human ligand for GPR119, the mice had an improved glucose tolerance when compared with control mice that were gavaged with E. coli harbouring an empty vector ${ }^{154}$. Finally, MECO-1, which is a bacterial melanocortin-like peptide that has structural similarities to $\alpha-\mathrm{MSH}$ and adrenocorticotropin $(\mathrm{ACTH})^{155}$, inhibits the release of cytokines when exposed to pro-inflammatory stimuli in vitro and rescues mice from death caused by endotoxins. Whether the microbial peptide also has endocrine functions like its human homologous hormones is unknown.

In summary, an overwhelming amount of data generated both in humans and in animals suggest important involvements of the primary saccharolytically derived microbial fermentation products SCFAs, succinate and ethanol in host metabolism. We know less about positive or undesired health implications of proteolytic or lipolytic fermentation by the gut microbiota (FIG. 1). An exception is the microbial fermentation of the dietary amino acids tryptophan and tyrosine that are metabolized to indoles and phenols, respectively ${ }^{156}$. In the liver, the compounds are converted to indoxyl sulfate and $p$-cresyl sulfate, the blood concentrations of which are elevated in CMD. In chronic kidney disease, elevated circulating levels of the two metabolites are associated with progression of chronic kidney disease, risk of CMD and all-cause mortality ${ }^{157}$. In addition, experiments in mice suggest that microbiota-produced gluconeogenic amino acid metabolites, including indoxyl sulfate, contribute to maintaining glucose homeostasis ${ }^{158}$.

\section{Conclusions and perspectives}

The past two decades of research within the microbiome field has made it evident that human biology means the biology of the holobiont Homo sapiens. In other words, human biology is contextual on the coexisting microorganisms, with the majority living in the digestive tract from where they produce or modify various chemicals, or trigger host reactions that affect various physiological functions including immunity, neurobiology and, not least, metabolism. As appears from our discussion of the pertinent literature on metabolism and the intestinal microbiota, the research field is still at a juvenile stage both in its basic and translational dimensions. With the focus on the influence of the gut microbiome on the overall functional metabolic read-outs, much still needs to be learned. The knowledge gaps to be filled include annotation of hundreds of as yet unknown chemical compounds in the metabolomes and peptidomes of various body fluids as well as approaches to decipher compounds of solely host, microbial and dietary origin, or of combined origin ${ }^{159}$. Most studies are snapshots of microbiome landscapes and more information is needed on the short-term and long-term dynamics of the intestinal microbiome. Importantly, very little of the novel knowledge is validated or has maturated to stages where it can be translated to guide personal everyday health behaviour (public health) or clinical practice. To achieve such goals, several conceptual and analytical challenges need attention in future studies where data on the microbiome are integrated with other omics read-out and bioclinical variables (BOX 2). 
The fact that the human intestinal microbiota is unique at deep resolution levels, with substantial structural variations in species and subspecies that influence microbiome functionality, is a major conceptual and statistical challenge when comparing individual microbiota irrespective of the health status of study subjects ${ }^{17}$. Moreover, there are several more technical and database-related shortcomings, including incomplete microbial genome databases and a lack of functional annotation of the majority of microbial genes. Similarly, knowledge about the regulation of transcriptional and translational activities of the gut microbiome is sparse, and when it comes to the interpretation of the outcome of untargeted metabolome profiling, which is often compared with microbiome features, the majority of read-outs are neither annotated nor quantified ${ }^{70}$.

When considering the translational implications of microbiome research, it recently became evident that most drugs influence microbiome, metatranscriptome and metabolome profiles in addition to their well-known effects on bioclinical variables ${ }^{5,62-64}$. Blindness to the impact of medication on 'omics' analyses may therefore have inflated the reported findings in human studies of any relationships between the gut microbiome and host metabolism, which we have discussed in this Review. Ideally, any such omics-based studies should be performed in drug-naive individuals. This demand is difficult to accommodate in studies of individuals with chronic metabolic diseases who often are prescribed multiple medications. Instead, more studies are currently undertaken in individuals at high risk of developing metabolic diseases and prior to medication, for example, in treatment-naive pre-morbid individuals. Yet another shortcoming of microbiome studies is the fact that microbiome analysis has so far largely been limited to relative abundances, failing therefore to account for biologically relevant differences between microbiomes owing to considerable sample variation in the bacterial cell density of stools or intestinal contents ${ }^{160}$.

Experimental studies of the targets and mechanisms of action of a given microbial compound are, for obvious reasons, done in vitro in assumed target tissues or cells and are complemented with in vivo studies in rodents (BOX 2). However, many experimental studies fall short owing to lack of appropriate test animals with a biology and behaviour close to those of humans and, especially when unravelling the role of the gut microbiome in disease causation, there is often a lack of animal models with genetic susceptibilities comparable to those of the human disorders under examination. Nevertheless, experimental studies are not exclusively done in cellular systems or animal models. In fact, clinically controlled trials are by their nature mechanistic experiments. Because of the massive and complex inter-individual variation in the gut microbiome, the ideal human trial design is to perform various controlled crossover interventions in the same individuals for years. Intra-individual trials have been done but are very resource demanding and have no potential for generalization to the population level ${ }^{161}$. Therefore, the only realistic way forward to gain population-relevant mechanistic insights into how the gut microbiome mediates or modifies the effects of diet, exercise, drugs and so forth in the human setting appears to be through carefully prepared randomized controlled trials of long duration. That means the inclusion of homogeneous groups of people who have undergone deep and extensive phenotyping in a sufficient number to have statistical power to address the hypothesis in focus.

Finally, the complexity of the gut microbiome is daunting, and the global intestinal microbiome is far more than bacteria and archaea. It also includes fungi, bacteriophages and eukaryotic virus. Future, sequencing-based and culture-based gut microorganism surveys combined with mechanistic exploitations of the gut bacteriome, archaeome, phageome, virome and mycobiome will exponentially expand our knowledge about the interactions within the global intestinal microbial community. Moreover, not least, exciting new knowledge about the multitude of chemicals that the global gut microbiome produces affecting host physiology and numerous pathologies may foster novel efficacious paths to stabilize metabolic health of the human holobiont and prevent or combat common human metabolic disorders.

\section{Published online 4 September 2020}

1. Lynch, S. V. \& Pedersen, O. The human intestinal microbiome in health and disease. N. Engl. J. Med. 375, 2369-2379 (2016)

2. Jaacks, L. M. et al. The obesity transition: stages of the global epidemic. Lancet Diabetes Endocrinol. 7, 231-240 (2019)

3. Younossi, Z. M. et al. Global epidemiology of nonalcoholic fatty liver disease - meta-analytic assessment of prevalence, incidence, and outcomes. Hepatology 64, 73-84 (2016).

4. Zheng, Y., Ley, S. H. \& Hu, F. B. Global aetiology and epidemiology of type 2 diabetes mellitus and its complications. Nat. Rev. Endocrinol. 14, 88 (2018).

5. Reddy, K. S. \& Yusuf, S. Emerging epidemic of cardiovascular disease in developing countries. Circulation 97, 596-601 (1998).

6. Lakka, H.-M. et al. The metabolic syndrome and total and cardiovascular disease mortality in middle-aged men. JAMA 288, 2709-2716 (2002).

7. Müller, O. \& Krawinkel, M. Malnutrition and health in developing countries. CMAJ 173, 279-286 (2005).

8. Qin, J. et al. A metagenome-wide association study of gut microbiota in type 2 diabetes. Nature 490 55-60 (2012).

This is the first metagenome-wide association study to identify links between the gut microbiome and T2D.
9. Qin, N. et al. Alterations of the human gut microbiome in liver cirrhosis. Nature 513, 59-64 (2014).

10. Koeth, R. A. et al. Intestinal microbiota metabolism of I-carnitine, a nutrient in red meat, promotes atherosclerosis. Nat. Med. 19, 576-585 (2013)

11. Allin, K. H. et al. Aberrant intestinal microbiota in individuals with prediabetes. Diabetologia 61 , 810-820 (2018)

12. Bäckhed, F. et al. The gut microbiota as an environmental factor that regulates fat storage. Proc. Natl Acad. Sci. USA 101, 15718-15723 (2004).

13. Qin, J. et al. A human gut microbial gene catalogue established by metagenomic sequencing. Nature $\mathbf{4 6 4}$ 59-65 (2010).

14. Pedersen, H. K. et al. Human gut microbes impact host serum metabolome and insulin sensitivity. Nature 535, 376-381 (2016). This study identifies $P$. copri and B. vulgatus as the main species driving the association between biosynthesis of BCAAs and insulin resistance under conditions of high fat intake.

15. Pedersen, H. K. et al. A computational framework to integrate high-throughput '-omics' datasets for the identification of potential mechanistic links. Nat. Protoc. 13, 2781 (2018).
16. Korem, T. et al. Growth dynamics of gut microbiota in health and disease inferred from single metagenomic samples. Science 349, 1101-1106 (2015).

17. Zeevi, D. et al. Structural variation in the gut microbiome associates with host health. Nature 568 43-48 (2019).

18. Huttenhower, C. et al. Structure, function and diversity of the healthy human microbiome. Nature $\mathbf{4 8 6}$, 207-214 (2012)

19. Rothschild, D. et al. Environment dominates over host genetics in shaping human gut microbiota. Nature 555, 210-215 (2018)

20. Falony, G., Vieira-Silva, S. \& Raes, J. Richness and ecosystem development across faecal snapshots of the gut microbiota. Nat. Microbiol. 3, 526-528 (2018).

21. Jakobsson, H. E. et al. Decreased gut microbiota diversity, delayed Bacteroidetes colonisation and reduced Th 1 responses in infants delivered by caesarean section. Gut 63, 559-566 (2014).

22. Rodriguez, J. M. et al. The composition of the gut microbiota throughout life, with an emphasis on early life. Microb. Ecol. Health Dis. 26, 26050 (2015).

23. Flint, H. J., Scott, K. P., Louis, P. \& Duncan, S. H. The role of the gut microbiota in nutrition and health. 
Nat. Rev. Gastroenterol. Hepatol. 9, 577-589 (2012).

24. Shafquat, A., Joice, R., Simmons, S. L. \& Huttenhower, C. Functional and phylogenetic assembly of microbial communities in the human microbiome. Trends Microbiol. 22, 261-266 (2014).

25. Clemente, J. C. et al. The microbiome of uncontacted Amerindians. Sci. Adv. 1, e1500183 (2015).

26. Smits, S. A. et al. Seasonal cycling in the gut microbiome of the Hadza hunter-gatherers of Tanzania. Science 357 802-806 (2017).

27. Tyakht, A. V. et al. Human gut microbiota community structures in urban and rural populations in Russia. Nat. Commun. 4, 1-9 (2013).

28. Le Chatelier, E. et al. Richness of human gut microbiome correlates with metabolic markers. Nature $\mathbf{5 0 0}$, 541-546 (2013).

This work shows that adults with low bacterial gene richness in their gut microbiome feature insulin resistance, pro-inflammation dyslipidaemia and increased body adiposity compared with individuals with high bacterial gene richness.

29. Cox, L. M. \& Blaser, M. J. Antibiotics in early life and obesity. Nat. Rev. Endocrinol. 11, 182-190 (2015)

30. Ajslev, T., Andersen, C., Gamborg, M., Sørensen, T. $\&$ Jess, T. Childhood overweight after establishment of the gut microbiota: the role of delivery mode, pre-pregnancy weight and early administration of antibiotics. Int. J. Obes. 35, 522 (2011).

31. Mor, A. et al. Prenatal exposure to systemic antibacterials and overweight and obesity in Danish schoolchildren: a prevalence study. Int. J. Obes. $\mathbf{3 9}$, 1450 (2015).

32. Palleja, A. et al. Recovery of gut microbiota of healthy adults following antibiotic exposure. Nat. Microbiol. 3 1255-1265 (2018)

33. Thuny, F. et al. Vancomycin treatment of infective endocarditis is linked with recently acquired obesity. PLOS ONE 5, e9074 (2010).

34. Mikkelsen, K. H. et al. Effect of antibiotics on gut microbiota, gut hormones and glucose metabolism. PLOS ONE 10, e0142352 (2015).

35. Reijnders, D. et al. Effects of gut microbiota manipulation by antibiotics on host metabolism in obese humans: a randomized double-blind placebo-controlled trial. Cell Metab. 24, 63-74 (2016)

36. Fujisaka, S. et al. Antibiotic effects on gut microbiota and metabolism are host dependent. J. Clin. Invest. 126, 4430-4443 (2016)

37. Yatsunenko, T. et al. Human gut microbiome viewed across age and geography. Nature 486, 222-227 (2012).

38. Cotillard, A. et al. Dietary intervention impact on gut microbial gene richness. Nature 500, 585-588 (2013).

39. World Health Organization. Obesity: Preventing and Managing the Global Epidemic (World Health Organization, 2000)

40. McAllister, E. J. et al. Ten putative contributors to the obesity epidemic. Crit. Rev. Food Sci. Nutr. 49 868-913 (2009).

41. Turnbaugh, P. J et al An obesity-associated gut microbiome with increased capacity for energy harvest. Nature 444, 1027-1031 (2006).

42. Tims, S. et al. Microbiota conservation and BMI signatures in adult monozygotic twins. ISME J. 7 707-717 (2013).

43. Gophna, U., Konikoff, T. \& Nielsen, H. B. Oscillospira and related bacteria-from metagenomic species to metabolic features. Environ. Microbiol. 19, 835-841 (2017)

44. Miller, T. L., Wolin, M., de Macario, E. C. \& Macario, A Isolation of Methanobrevibacter smithii from human feces. Appl. Environ. Microbiol. 43, 227-232 (1982).

45. Liu, R. et al. Gut microbiome and serum metabolome alterations in obesity and after weight-loss intervention. Nat. Med. 23, 859-868 (2017)

This study links intestinal microbiota alterations, circulating amino acids and obesity, and suggests that a possible way to intervene in obesity is by targeting the gut microbiota.

46. Thingholm, L. B. et al. Obese individuals with and without type 2 diabetes show different gut microbial functional capacity and composition. Cell Host Microbe 26, 252-264.e10 (2019).

47. Ridaura, V. K. et al. Gut microbiota from twins discordant for obesity modulate metabolism in mice. Science 341, 1241214 (2013).

This study shows that adiposity is transmissible in a diet-dependent manner from human to mouse and is associated with alterations in serum levels of BCAAs.
48. Vieira-Silva, S. et al. Statin therapy is associated with lower prevalence of gut microbiota dysbiosis. Nature 581, 310-315 (2020).

This study observes that obesity-associated microbiota dysbiosis is negatively associated with statin treatment, indicating statins as a possible target for the development of future drug-based strategies for the modulation of the intestinal microbiota.

49. Jensen, A. B. et al. Increase in clinically recorded type 2 diabetes after colectomy. eLife 7, e37420 (2018).

50. Thaiss, C. A. et al. Hyperglycemia drives intestinal barrier dysfunction and risk for enteric infection. Science 359, 1376-1383 (2018).

51. Association, A. D. Diagnosis and classification of diabetes mellitus. Diabetes Care 37, S81-S90 (2014).

52. Deshpande, A. D., Harris-Hayes, M. \& Schootman, M. Epidemiology of diabetes and diabetes-related complications. Phys. Ther. 88, 1254-1264 (2008)

53. Grarup, N., Sandholt, C. H., Hansen, T. \& Pedersen, O. Genetic susceptibility to type 2 diabetes and obesity: from genome-wide association studies to rare variants and beyond. Diabetologia 57, 1528-1541 (2014).

54. Kasuga, M. Insulin resistance and pancreatic $\beta$ cell failure. J. Clin. Invest. 116, 1756-1760 (2006)

55. Bornfeldt, K. E. \& Tabas, I. Insulin resistance, hyperglycemia, and atherosclerosis. Cell Metab. 14 575-585 (2011).

56. Laakso, M. \& Kuusisto, J. Insulin resistance and hyperglycaemia in cardiovascular disease development. Nat. Rev. Endocrinol. 10, 293-302 (2014).

57. Vila, A. V. et al. Impact of commonly used drugs on the composition and metabolic function of the gut microbiota. Nat. Commun. 11, 1-11 (2020).

58. Zhong, $\mathrm{H}$. et al. Distinct gut metagenomics and metaproteomics signatures in prediabetics and treatment-naïve type 2 diabetics. EBioMedicine 47 , 373-383 (2019)

59. Whiting, D. R., Guariguata, L., Weil, C. \& Shaw, J. IDF diabetes atlas: global estimates of the prevalence of diabetes for 2011 and 2030. Diabetes Res. Clin. Pract. 94, 311-321 (2011).

60. Crusell, M. K. W. et al. Gestational diabetes is associated with change in the gut microbiota composition in third trimester of pregnancy and postpartum. Microbiome 6, 89 (2018).

61. Karlsson, F. H. et al. Gut metagenome in European women with normal, impaired and diabetic glucose control. Nature 498, 99-103 (2013)

62. Forslund, K. et al. Disentangling type 2 diabetes and metformin treatment signatures in the human gut microbiota. Nature 528, 262-266 (2015).

This work outlines a paradigm to disentangle disease microbiome features from secondary changes in the microbiome induced by medication.

63. $\mathrm{Wu}, \mathrm{H}$. et al. Metformin alters the gut microbiome of individuals with treatment-naive type 2 diabetes, contributing to the therapeutic effects of the drug. Nat. Med. 23, 850-858 (2017).

64. Bryrup, T. et al. Metformin-induced changes of the gut microbiota in healthy young men: results of a nonblinded, one-armed intervention study. Diabetologia 62, 1024-1035 (2019)

65. Sun, L. et al. Gut microbiota and intestinal FXR mediate the clinical benefits of metformin. Nat. Med. 24, 1919 (2018)

66. De La Cuesta-Zuluaga, J. et al. Metformin is associated with higher relative abundance of mucindegrading Akkermansia muciniphila and several short-chain fatty acid-producing microbiota in the gut. Diabetes Care 40, 54-62 (2017).

67. Adeshirlarijaney, A , Zou, J., Tran, H. O., Chassaing, B. $\Sigma$ Gewirtz, A. T. Amelioration of metabolic syndrome by metformin associates with reduced indices of low-grade inflammation independently of the gut microbiota. Am. J. Physiol. Endocrinol. Metab. 317 , E1121-E1130 (2019)

68. Pryor, R. et al. Host-microbe-drug-nutrient screen identifies bacterial effectors of metformin therapy. Cell 178, 1299-1312.e29 (2019).

69. Herrington, W., Lacey, B., Sherliker, P., Armitage, J. $\Sigma$ Lewington, S. Epidemiology of atherosclerosis and the potential to reduce the global burden of atherothrombotic disease. Circ. Res. 118, 535-546 (2016).

70. Fan, Y. et al. Comprehensive metabolomic characterization of coronary artery diseases. J. Am. Coll. Cardiol. 68, 1281-1293 (2016)

71. Michos, E. D., McEvoy, J. W. \& Blumenthal, R. S. Lipid management for the prevention of atherosclerotic cardiovascular disease. N. Engl. J. Med. 381 1557-1567 (2019).
72. Hansson, G. K. Inflammation, atherosclerosis, and coronary artery disease. N. Engl. J. Med. 352 1685-1695 (2005).

73. Jie, Z. et al. The gut microbiome in atherosclerotic cardiovascular disease. Nat. Commun. 8, 845 (2017)

74. Sokol, $\mathrm{H}$. et al. Faecalibacterium prausnitzii is an antiinflammatory commensal bacterium identified by gut microbiota analysis of Crohn disease patients. Proc. Natl Acad. Sci. USA 105, 16731-16736 (2008).

75. Cui, X. et al. Metagenomic and metabolomic analyses unveil dysbiosis of gut microbiota in chronic heart failure patients. Sci. Rep. 8, 1-15 (2018).

76. Wang, Z. et al. Gut flora metabolism of phosphatidylcholine promotes cardiovascular disease. Nature 472, 57-63 (2011).

This work discovers a relationship between the gut microbiota-dependent metabolism of dietary phosphatidylcholine and pathogenesis of arteriosclerosis.

77. Zhu, W. et al. Gut microbial metabolite TMAO enhances platelet hyperreactivity and thrombosis risk. Cell 165, 111-124 (2016).

78. Wang, Z. et al. Non-lethal inhibition of gut microbial trimethylamine production for the treatment of atherosclerosis. Cell 163, 1585-1595 (2015). This study discovers a structural analogue of choline that inhibits microbial TMA lyases and the production of TMA by the gut microbiota.

79. Haghikia, A. et al. Gut microbiota-dependent trimethylamine $\mathrm{N}$-oxide predicts risk of cardiovascular events in patients with stroke and is related to proinflammatory monocytes. Arterioscl. Throm. Vas. Biol. 38, 2225-2235 (2018).

80. Senthong, V. et al. Intestinal microbiota-generated metabolite trimethylamine- $\mathrm{N}$-oxide and 5-year mortality risk in stable coronary artery disease: the contributory role of intestinal microbiota in a COURAGE-like patient cohort. J. Am. Heart Assoc. 5 e002816 (2016)

81. Collins, H. L. et al. I-Carnitine intake and high trimethylamine $\mathrm{N}$-oxide plasma levels correlate with low aortic lesions in $\mathrm{ApoE}^{-/-}$transgenic mice expressing CETP. Atherosclerosis 244, 29-37 (2016)

82. He, K. et al. Accumulated evidence on fish consumption and coronary heart disease mortality: a meta-analysis of cohort studies. Circulation 109, 2705-2711 (2004).

83. Koay, Y. C. et al. Plasma levels of TMAO can be increased with 'healthy' and 'unhealthy' diets and do not correlate with the extent of atherosclerosis but with plaque instability. Cardiovasc. Res. 8, cvaa094 (2020).

84. Tang, W. W. et al. Intestinal microbial metabolism of phosphatidylcholine and cardiovascular risk. N. Engl. J. Med. 368, 1575-1584 (2013).

85. Brunt, E. M. et al. Nonalcoholic fatty liver disease (NAFLD) activity score and the histopathologic diagnosis in NAFLD: distinct clinicopathologic meanings. Hepatology 53, 810-820 (2011).

86. Jiang, W. et al. Dysbiosis gut microbiota associated with inflammation and impaired mucosal immune function in intestine of humans with non-alcoholic fatty liver disease. Sci. Rep. 5, 8096 (2015).

87. Zhu, L. et al. Characterization of gut microbiomes in nonalcoholic steatohepatitis (NASH) patients: a connection between endogenous alcohol and NASH Hepatology 57, 601-609 (2013).

88. Del Chierico, F. et al. Gut microbiota profiling of pediatric nonalcoholic fatty liver disease and obese patients unveiled by an integrated meta-omics-based approach. Hepatology 65, 451-464 (2017).

89. Nair, S., Cope, K., Terence, R. H. \& Diehl, A. M Obesity and female gender increase breath ethanol concentration: potential implications for the pathogenesis of nonalcoholic steatohepatitis. Am. J. Gastroenterol. 96, 1200-1204 (2001)

90. Rao, R., Seth, A. \& Sheth, P. Recent advances in alcoholic liver disease I. Role of intestinal permeability and endotoxemia in alcoholic liver disease. $\mathrm{Am}$. $\mathrm{J}$. Physiol. Gastrointest. Liver Physiol. 286, G881-G884 (2004).

91. Xu, J. et al. Synergistic steatohepatitis by moderate obesity and alcohol in mice despite increased adiponectin and p-AMPK. J. Hepatol. 55, 673-682 (2011).

92. de Medeiros, I. C. \& de Lima, J. G. Is nonalcoholic fatty liver disease an endogenous alcoholic fatty liver disease? - A mechanistic hypothesis. Med. Hypotheses 85, 148-152 (2015).

93. Schwenger, K. J., Clermont-Dejean, N. \& Allard, J. P. The role of the gut microbiome in chronic liver disease: the clinical evidence revised. JHEP Rep. 1 , 214-226 (2019). 
94. Le Roy, T et al. Intestinal microbiota determines development of non-alcoholic fatty liver disease in mice. Gut 62, 1787-1794 (2013).

95. Yuan, J. et al. Fatty liver disease caused by highalcohol-producing Klebsiella pneumoniae. Cell Metab. 30, 675-688.e7 (2019)

96. Hoyles, L. et al. Molecular phenomics and metagenomics of hepatic steatosis in non-diabetic obese women. Nat. Med. 24, 1070-1080 (2018) This study demonstrates that individuals with liver steatosis have low microbial gene richness and increased genetic potential for the processing of dietary lipids and endotoxin biosynthesis, hepatic inflammation and dysregulation of aromatic and BCAA metabolism.

97. UNICEF-WHO-The World Bank Group: Joint Child Malnutrition Estimates - levels and trends in child malnutrition: key findings of the 2015 edition. Global Database on Child Growth and Malnutrition (WHO, 2015)

98. Black, R. E. et al. Maternal and child undernutrition global and regional exposures and health consequences. Lancet 371, 243-260 (2008)

99. Million, M., Diallo, A. \& Raoult, D. Gut microbiota and malnutrition. Microb. Pathog. 106, 127-138 (2017).

100. Smith, M. I. et al. Gut microbiomes of Malawian twin pairs discordant for kwashiorkor. Science 339 548-554 (2013).

101. Lee, J.-H., Li, X. \& O'Sullivan, D. J. Transcription analysis of a lantibiotic gene cluster from Bifidobacterium longum DJO10A. Appl. Environ. Microbiol. 77, 5879-5887 (2011).

102. Mata, L. J. et al. Gastrointestinal flora of children with protein - calorie malnutrition. Am. J. Clin. Nutr. 25 1118-1126 (1972)

103. Kelly, D. et al. Commensal anaerobic gut bacteria attenuate inflammation by regulating nuclearcytoplasmic shuttling of PPAR- $\gamma$ and RelA. Nat. Immunol. 5, 104-112 (2004)

104. Martens, J.-H., Barg, H., Warren, M. A. \& Jahn, D. Microbial production of vitamin B 12. Appl. Microbiol. Biotechnol. 58, 275-285 (2002).

105. Stecher, B. \& Hardt, W.-D. The role of microbiota in infectious disease. Trends Microbiol. 16, 107-114 (2008).

106. Gehrig, J. L. et al. Effects of microbiota-directed foods in gnotobiotic animals and undernourished children. Science 365, eaau4732 (2019).

107. Jumpertz, R. et al. Energy-balance studies reveal associations between gut microbes, caloric load, and nutrient absorption in humans. Am. J. Clin. Nutr. 94 58-65 (2011).

108. Lin, H. V. et al. Butyrate and propionate protect against diet-induced obesity and regulate gut hormones via free fatty acid receptor 3 -independent mechanisms. PLoS ONE 7, e35240 (2012).

109. Xiong, Y. et al. Short-chain fatty acids stimulate leptin production in adipocytes through the G proteincoupled receptor GPR41. Proc. Natl Acad. Sci. USA 101, 1045-1050 (2004).

110. Vinolo, M. A. R. et al. Tributyrin attenuates obesityassociated inflammation and insulin resistance in highfat-fed mice. Am. J. Physiol. Endocrinol. Metab. 303 E272-E282 (2012)

111. Maslowski, K. M. et al. Regulation of inflammatory responses by gut microbiota and chemoattractant receptor GPR43. Nature 461, 1282-1286 (2009).

112. Macia, L. et al. Metabolite-sensing receptors GPR43 and GPR 109A facilitate dietary fibre-induced gut homeostasis through regulation of the inflammasome. Nat. Commun. 6, 6734 (2015)

113. Pluznick, J. L. Renal and cardiovascular sensory receptors and blood pressure regulation. $A m$. Physiol. Ren. Physiol. 305, F439-F444 (2013).

114. Byndloss, M. X. et al. Microbiota-activated PPAR- $\gamma$ signaling inhibits dysbiotic Enterobacteriacea expansion. Science 357, 570-575 (2017).

115. Tang, T. W. et al. Loss of gut microbiota alters immune system composition and cripples postinfarction cardiac repair. Circulation 139, 647-659 (2019).

116. Chambers, E. S. et al. Effects of targeted delivery of propionate to the human colon on appetite regulation, body weight maintenance and adiposity in overweight adults. Gut 64, 1744-1754 (2015)

117. Sanna, S. et al. Causal relationships among the gut microbiome, short-chain fatty acids and metabolic diseases. Nat. Genet. 51, 600 (2019).

This study presents data providing evidence of a causal effect of the gut microbiome on metabolic traits

118. Tirosh, A. et al. The short-chain fatty acid propionate increases glucagon and FABP4 production, impairing insulin action in mice and humans. Sci. Trans/ Med. 11, eaav0120 (2019).

119. Bouter, K. et al. Differential metabolic effects of ora butyrate treatment in lean versus metabolic syndrome subjects. Clin. Trans. Gastroenterol 9, 155 (2018).

120. Gao, X. et al. Acetate functions as an epigenetic metabolite to promote lipid synthesis under hypoxia. Nat. Commun. 7, 11960 (2016)

121. Perry, R. J. et al. Acetate mediates a microbiomebrain- $\beta$-cell axis to promote metabolic syndrome. Nature 534, 213-217 (2016)

122. Freeland, K. R. \& Wolever, T. M. Acute effects of intravenous and rectal acetate on glucagon-like peptide-1, peptide YY, ghrelin, adiponectin and tumour necrosis factor- $\alpha$. Br. J. Nutr. 103, 460-466 (2010).

123. Ridlon, J. M., Kang, D.-J. \& Hylemon, P. B. Bile salt biotransformations by human intestinal bacteria. J. Lipid Res. 47, 241-259 (2006).

124. Fiorucci, S., Mencarelli, A., Palladino, G. \& Cipriani, S Bile-acid-activated receptors: targeting TGR5 and farnesoid-X-receptor in lipid and glucose disorders. Trends Pharmacol. Sci. 30, 570-580 (2009).

125. Prawitt, J. et al. Farnesoid X receptor deficiency improves glucose homeostasis in mouse models of obesity. Diabetes 60, 1861-1871 (2011).

126. Thomas, C. et al. TGR5-mediated bile acid sensing controls glucose homeostasis. Cell Metab. 10, 167-177 (2009)

127. Makishima, M. et al. Identification of a nuclear receptor for bile acids. Science 284, 1362-1365 (1999).

128. Kawamata, Y. et al. A G protein-coupled receptor responsive to bile acids. J. Biol. Chem. 278, 9435-9440 (2003).

129. Pathak, P. et al. Intestine farnesoid X receptor agonist and the gut microbiota activate G-protein bile acid receptor-1 signaling to improve metabolism. Hepatology 68, 1574-1588 (2018).

130. Modica, S., Gadaleta, R. M. \& Moschetta, A Deciphering the nuclear bile acid receptor FXR paradigm $\mathrm{NuCl}$ Recept Signal. https://doi.org 10.1621/nrs.08005 (2010).

131. Spinelli, V. et al. Influence of Roux-en-Y gastric bypass on plasma bile acid profiles: a comparative study between rats, pigs and humans. Int. J. Obes. 40, 1260 (2016).

132. Kindel, T. L. et al. Increased glycine-amidated hyocholic acid correlates to improved early weight loss after sleeve gastrectomy. Surg. Endosc. 32, 805-812 (2018).

133. Canfora, E. E., Meex, R. C., Venema, K. \& Blaak, E. E. Gut microbial metabolites in obesity, NAFLD and T2DM. Nat. Rev. Endocrinol. 15, 261-273 (2019).

134. Bansal, T., Alaniz, R. C., Wood, T. K. \& Jayaraman, A The bacterial signal indole increases epithelial-cell tight-junction resistance and attenuates indicators of inflammation. Proc. Natl Acad. Sci. USA 107 228-233 (2010)

135. Shimada, Y. et al. Commensal bacteria-dependent indole production enhances epithelial barrier function in the colon. PLoS ONE 8, e80604 (2013).

136. Chimerel, $C$. et al. Bacterial metabolite indole modulates incretin secretion from intestinal enteroendocrine L cells. Cell Rep. 9, 1202-1208 (2014).

137. De Mello, V. D. et al. Indolepropionic acid and novel lipid metabolites are associated with a lower risk of type 2 diabetes in the Finnish Diabetes Prevention Study. Sci. Rep. 7, 46337 (2017)

This study shows that microbial indole propionate and additional metabolites associate with lower risk of incident T2D.

138. Wang, T. J. et al. Metabolite profiles and the risk of developing diabetes. Nat. Med. 17, 448 (2011).

139. Sun, H. et al. Catabolic defect of branched-chain amino acids promotes heart failure. Circulation 133 2038-2049 (2016).

140. Koh, A. et al. Microbially produced imidazole propionate impairs insulin signaling through $\mathrm{mTORC} 1$ Cell 175, 947-961.e17 (2018).

This study demonstrates that imidazole propionate is produced from histidine in a gut simulator at higher concentrations when using faecal microbiota from subjects with T2D than from individuals without T2D, and that it impairs glucose tolerance when administered to mice.

141. MacDonald, M. J., Fahien, L. A., Mertz, R. J. \& Rana, R. S. Effect of esters of succinic acid and other citric acid cycle intermediates on insulin release and inositol phosphate formation by pancreatic islets. Arch. Biochem. Biophys. 269, 400-406 (1989).
142. Mills, E. L. et al. Accumulation of succinate controls activation of adipose tissue thermogenesis. Nature $560,102-106$ (2018)

143. Tannahill, G. et al. Succinate is an inflammatory signa that induces IL-1 $\beta$ through HIF-1 $\alpha$. Nature 496, 238-242 (2013).

144. Suárez-Zamorano, N. et al. Microbiota depletion promotes browning of white adipose tissue and reduces obesity. Nat. Med. 21, 1497 (2015).

145. Li, B. et al. Microbiota depletion impairs thermogenesis of brown adipose tissue and browning of white adipose tissue. Cell Rep. 26, 2720-2737.e5 (2019).

146. Kamio Y \& Nikaido H Outer membrane of Salmonella typhimurium: accessibility of phospholipid head groups to phospholipase $\mathrm{C}$ and cyanogen bromide activated dextran in the external medium Biochemistry 15, 2561-2570 (1976).

147. Cani, P. D. et al. Metabolic endotoxemia initiates obesity and insulin resistance. Diabetes $\mathbf{5 6}$, 1761-1772 (2007).

148. Luck, H. et al. Gut-associated lgA ${ }^{+}$immune cells regulate obesity-related insulin resistance. Nat. Commun. 10 1-17 (2019).

149. Winer, D. A., Luck, H., Tsai, S. \& Winer, S. The intestinal immune system in obesity and insulin resistance. Cell Metab. 23, 413-426 (2016).

150. Breton, J. et al. Gut commensal E. coli proteins activate host satiety pathways following nutrientinduced bacterial growth. Cell Metab. 23, 324-334 (2016).

151. Tennoune, N. et al. Bacterial ClpB heat-shock protein, an antigen-mimetic of the anorexigenic peptide $\alpha-\mathrm{MSH}$ at the origin of eating disorders. Transl Psychiat. 4, e458-e458 (2014)

152. Breton, J. et al. Elevated plasma concentrations of bacterial $\mathrm{ClpB}$ protein in patients with eating disorders. Int. J. Eat. Disord. 49, 805-808 (2016).

153. Cohen, L. J. et al. Functional metagenomic discovery of bacterial effectors in the human microbiome and isolation of commendamide, a GPCR G2A/132 agonist. Proc. Natl Acad. Sci. USA 112, E4825-E4834 (2015).

154. Cohen, L. J. et al. Commensal bacteria make GPCR ligands that mimic human signalling molecules. Nature 549, 48 (2017).

155. Qiang, X. et al. New melanocortin-like peptide of E. coli can suppress inflammation via the mammalian melanocortin-1 receptor (MC1R): possible endocrinelike function for microbes of the gut. NPJ Biofilms Microbiomes 3, 31 (2017).

156. Elsden, S. R., Hilton, M. G. \& Waller, J. M. The end products of the metabolism of aromatic amino acids by Clostridia. Arch. Microbiol. 107 283-288 (1976)

157. Lin, C.-J., Wu, V., Wu, P.-C. \& Wu, C.-J. Meta-analysis of the associations of $p$-cresyl sulfate (PCS) and indoxyl sulfate (IS) with cardiovascular events and all-cause mortality in patients with chronic renal failure. PLOS ONE 10, e0132589 (2015).

158. Krisko, T. I. et al. Dissociation of adaptive thermogenesis from glucose homeostasis in microbiome-deficient mice. Cell Metab. 31, 592-604.e9 (2020).

159. Vojinovic, D. et al. Relationship between gut microbiota and circulating metabolites in population-based cohorts. Nat. Commun. 10, 1-7 (2019)

160. Vandeputte, D. et al. Quantitative microbiome profiling links gut community variation to microbial load. Nature 551, 507 (2017).

This study builds a workflow for the quantitative microbiome profiling of faecal material, showing that quantitative microbiome profiling has a substantial effect on co-occurrence analyses and the characterization of disease-associated microbiota perturbations.

161. Zhou, W. et al. Longitudinal multi-omics of host-microbe dynamics in prediabetes. Nature 569, 663-671 (2019).

162. Flint, A., Raben, A., Rehfeld, J., Holst, J. \& Astrup, A The effect of glucagon-like peptide- 1 on energy expenditure and substrate metabolism in humans. Int. J. Obes. 24, 288 (2000)

163. Batterham, R. L. et al. Gut hormone PYY 3-36 physiologically inhibits food intake. Nature 418, 650 (2002).

164. Holz, I. V. IV, G. G., Kiihtreiber, W. M. \& Habener, J. F. Pancreatic $\beta$-cells are rendered glucose-competent by the insulinotropic hormone glucagon-like peptide-1 (7-37). Nature 361, 362 (1993).

165. Macfarlane, S. \& Macfarlane, G. T. Composition and metabolic activities of bacterial biofilms colonizing food residues in the human gut. Appl. Environ. Microbiol. 72, 6204-6211 (2006). 
166. Roager, H. M. et al. Colonic transit time is related to bacterial metabolism and mucosal turnover in the gut. Nat. Microbiol. 1, 16093 (2016). This study indicates that the colonic transit time is an important factor to consider in microbiome and metabolomics studies.

167. Russell, W. R. et al. Major phenylpropanoid-derived metabolites in the human gut can arise from microbial fermentation of protein. Mol. Nutr. Food Res. 57, 523-535 (2013).

168. Flemer, B. et al. The oral microbiota in colorectal cance is distinctive and predictive. Gut 67, 1454-1463 (2018).

169. Plaza Oñate, F. et al. MSPminer: abundance-based reconstitution of microbial pan-genomes from shotgun metagenomic data. Bioinformatics 35, 1544-1552 (2018).

170. Truong, D. T., Tett, A., Pasolli, E., Huttenhower, C. \& Segata, N. Microbial strain-level population structure and genetic diversity from metagenomes. Genome Res. 27, 626-638 (2017).

171. Zolfo, M., Tett, A., Jousson, O., Donati, C. \& Segata, N. MetaMLST: multi-locus strain-level bacterial typing from metagenomic samples. Nucleic Acids Res. 45, e7-e7 (2016).

172. Truong, D. T. et al. MetaPhIAn2 for enhanced metagenomic taxonomic profiling. Nat Methods 12 902 (2015).

173. Greenblum, S., Carr, R. \& Borenstein, E. Extensive strain-level copy-number variation across human gut microbiome species. Cell 160, 583-594 (2015).

174. Langfelder, P. \& Horvath, S. WGCNA: an R package for weighted correlation network analysis. BMC Bioinforma. 9, 559 (2008)

175. Wikoff, W. R. et al. Diacetylspermine is a novel prediagnostic serum biomarker for non-small-cell lung cancer and has additive performance with pro-surfactant protein B. J. Clin. Oncol. 33, 3880 (2015).

176. de Hoffmann, E. Tandem mass spectrometry: a primer. J. Mass. Spectrom. 31, 129-137 (1996).

177. Donia, M. S. \& Fischbach, M. A. Small molecules from the human microbiota. Science 349, 1254766 (2015).

178. Cani, P. D. et al. Changes in gut microbiota control metabolic endotoxemia-induced inflammation in high-fat diet-induced obesity and diabetes in mice. Diabetes 57, 1470-1481 (2008)

179. Wolters, M. et al. Dietary fat, the gut microbiota, and metabolic health - a systematic review conducted within the MyNewGut project. Clin. Nutr. 38 , 2504-2520 (2018)

180. Kjølbæk, L. et al. Arabinoxylan oligosaccharides and polyunsaturated fatty acid effects on gut microbiota and metabolic markers in overweight individuals with signs of metabolic syndrome: a randomized cross-over trial. Clin. Nutr. 39, 67-79 (2019).

181. Dieterich, W. et al. Influence of low FODMAP and gluten-free diets on disease activity and intestinal microbiota in patients with non-celiac gluten sensitivity. Clin. Nutr. 38, 697-707 (2019).

182. Hansen, L. B. et al. A low-gluten diet induces changes in the intestinal microbiome of healthy Danish adults. Nat. Commun. 9, 4630 (2018)

183. Roager, H. M. et al. Whole grain-rich diet reduces body weight and systemic low-grade inflammation without inducing major changes of the gut microbiome: a randomised cross-over trial. Gut 68, 83-93 (2019).

184. Sanders, M. E., Merenstein, D. J., Reid, G., Gibson, G. R. $\&$ Rastall, R. A. Probiotics and prebiotics in intestinal health and disease: from biology to the clinic. Nat. Rev. Gastroenterol. Hepatol. 16, 605-616 (2019).

185. Martín, R. et al. Functional characterization of novel Faecalibacterium prausnitzii strains isolated from healthy volunteers: a step forward in the use of $F$. prausnitzii as a next-generation probiotic. Front. Microbiol. 8, 1226 (2017).

186. Depommier, C. et al. Supplementation with Akkermansia muciniphila in overweight and obese human volunteers: a proof-of-concept exploratory study. Nat. Med. 25, 1096-1103 (2019). This proof-of-concept study shows that supplementation with $A$. muciniphila improves several dysmetabolic features.

187. Cani, P. D. \& Van Hul, M. Novel opportunities for next-generation probiotics targeting metabolic syndrome. Curr. Opin. Biotechnol. 32, 21-27 (2015).

188. Kristensen, N. B. et al. Alterations in fecal microbiota composition by probiotic supplementation in healthy adults: a systematic review of randomized controlled trials. Genome Med. 8, 52 (2016).

189. Zmora, N. et al. Personalized gut mucosal colonization resistance to empiric probiotics is associated with unique host and microbiome features. Cell 174 . 1388-1405.e21 (2018).

190. Suez, J. et al. Post-antibiotic gut mucosal microbiome reconstitution is impaired by probiotics and improved by autologous FMT. Cell 174, 1406-1423.e16 (2018).

191. Everard, A. et al. Responses of gut microbiota and glucose and lipid metabolism to prebiotics in genetic obese and diet-induced leptin-resistant mice. Diabetes 60, 2775-2786 (2011)

192. Dewulf, E. M. et al. Insight into the prebiotic concept: lessons from an exploratory, double blind intervention study with inulin-type fructans in obese women. Gut 62, 1112-1121 (2013)
193. Nicolucci, A. C. et al. Prebiotics reduce body fat and alter intestinal microbiota in children who are overweight or with obesity. Gastroenterology 153 , 711-722 (2017).

194. Tsilingiri, K. et al. Probiotic and postbiotic activity in health and disease: comparison on a novel polarised ex vivo organ culture model. Gut 61, 1007-1015 (2012).

195. Plovier, H. et al. A purified membrane protein from Akkermansia muciniphila or the pasteurized bacterium improves metabolism in obese and diabetic mice. Nat. Med. 23, 107 (2017).

196. Van Nood, E. et al. Duodenal infusion of donor feces for recurrent Clostridium difficile. N. Engl. J. Med. 368, 407-415 (2013).

197. Kootte, R. S. et al. Improvement of insulin sensitivity after lean donor feces in metabolic syndrome is driven by baseline intestinal microbiota composition. Cell Metab. 26, 611-619.e6 (2017).

198. Duan, F. F., Liu, J. H. \& March, J. C. Engineered commensal bacteria reprogram intestinal cells into glucose-responsive insulin-secreting cells for the treatment of diabetes. Diabetes 64, 1794-1803 (2015).

199. Górski, A. et al. Perspectives of phage therapy in nonbacterial infections. Front. Microbiol. https://doi.org/ 10.3389/fmicb.2018.03306 (2018).

200. Ramachandran, G \& Bikard D. Editing the microbiome the CRISPR way. Philos. Trans. R. Soc. Lond. B Biol. Sci. 374, 20180103 (2019)

\section{Acknowledgements}

This study was supported by Marie Skłodowska-Curie Individual Fellowship 797267 (granted to Y.F.). The Novo Nordisk Foundation Center for Basic Metabolic Research is an independent research centre at the University of Copenhagen partially funded by an unrestricted donation from the Novo Nordisk Foundation.

\section{Author contributions}

The authors contributed equally to all aspects of the article

\section{Competing interests}

The authors declare no competing interests.

\section{Peer review information}

Nature Reviews Microbiology thanks M. Nieuwdorp, C. Thaiss and the other, anonymous, reviewer(s) for their contribution to the peer review of this work.

\section{Publisher's note}

Springer Nature remains neutral with regard to jurisdictional claims in published maps and institutional affiliations.

(c) Springer Nature Limited 2020 